\title{
Comparing PaaS offerings in light of SaaS development
} A comparison of PaaS platforms based on a practical case study

\author{
Stefan Walraven · Eddy Truyen · Wouter \\ Joosen
}

Received: date / Accepted: date

\begin{abstract}
Software vendors increasingly aim to apply the Software-as-a-Service (SaaS) delivery model instead of the traditional on-premise model. Platforms-as-a-Service (PaaS), such as Google App Engine and Windows Azure, deliver a computing platform and solution stack as a service, but they also aim to facilitate the development of cloud applications (SaaS). Such PaaS offerings should enable third parties to build and deliver multi-tenant SaaS applications while shielding the complexity of the underpinning middleware and infrastructure. This paper compares, on the basis of a practical case study, three different and representative PaaS platforms with respect to their support for SaaS application development. We have reengineered an on-premise enterprise application into a SaaS application and we have subsequently deployed it in three PaaS-based cloud environments. We have investigated the following qualities of the PaaS platforms from the perspective of SaaS development: portability of the application code base, available support for creating and managing multi-tenantaware applications, and quality of the tool support.
\end{abstract}

Keywords Cloud computing $\cdot$ PaaS comparison $\cdot$ SaaS applications $\cdot$ Development support

\section{Introduction}

Cloud computing is a trend that refers to the delivery of ICT solutions as online services, covering software applications, system software, hardware infrastructure, etc. The concept of cloud computing includes three cloud service delivery models [43, 79]:

- Infrastructure-as-a-Service (IaaS) is the delivery of fundamental computing resources (e.g. processing, storage and networks) as a service, for example Amazon EC2 [1]

Stefan Walraven · Eddy Truyen · Wouter Joosen

iMinds-DistriNet, KU Leuven, Celestijnenlaan 200A (postbox 2402), 3001 Leuven, Belgium

Tel.: +32 16 327819, Fax: +3216327996

E-mail: stefan.walraven@cs.kuleuven.be 
- Platform-as-a-Service (PaaS) provides a computing platform and solution stack upon which applications and services can be developed and hosted by using programming concepts and tools supported by the provider. Examples include Force.com [67,82] and Google App Engine [32].

- Software-as-a-Service (SaaS) is a software deployment model that delivers software applications as online, on-demand services, for example the Salesforce CRM application [68].

Software vendors increasingly deliver their software applications as online services, in accordance with the above-mentioned SaaS model, and thus become SaaS providers. This enables their customers to use these software services on demand. Customers are thus freed from the management of the underpinning middleware and infrastructure. But the SaaS model requires software vendors to harvest full benefit from economies of scale. Resources could thus be employed more efficiently and maintenance efforts can be centralized. These benefits, if well-achieved by the SaaS provider, add value to the offering, and reduce the operational costs of providing the software services.

One of the key enablers to leverage economies of scale is multi-tenancy [19, 36]. Typically, a service provider manages one dedicated application instance per customer (i.e. single-tenancy). We focus on the particular case of application-level multi-tenancy. In this context, we define multi-tenancy as an architectural style that enables service providers to serve end users from different tenants (i.e. customer organizations and companies) simultaneously by a single application instance on top of shared hardware and software infrastructure [36]. Besides the benefits of economies of scale, a multi-tenant architecture simplifies administration and provisioning of tenants, for example upgrades of the application only have to be applied to the shared instance and all tenants will automatically use the most recent version.

The decision to apply multi-tenancy for a SaaS application depends on the application- and tenant-specific requirements. Often this is a trade-off between cost efficiency and flexibility [81]. With multi-tenancy a higher level of sharing can be achieved, which leads to higher scalability and maximal resource utilization. However, it also introduces additional complexity for the SaaS developers (i) to ensure the necessary isolation between the different tenants [36], (ii) to offer a customizable application in order to meet the unique requirements of the different tenants [78], and (iii) to manage and monitor the different tenants in a fine-grained way.

PaaS tries to solve the challenges sketched above by delivering a higher-level computing platform and software stack, which aims to facilitate the development and deployment of cloud applications while also managing availability, elastic scalability, load balancing and other infrastructure-related concerns. PaaS can therefore play a major role for software vendors in the adoption of the cloud computing paradigm. If such a combination of a scalable platform and a full software stack can be provided, it would reduce the entrance barrier for software vendors to offer their respective capabilities to a wide market with a minimum of entry costs and infrastructure requirements [70]. Most recent research has focused on IaaS (e.g. Eucalyptus [55] and OpenNebula [77]), which allows software vendors to deploy and run arbitrary software on top of a virtualized infrastructure [43]. However, the maintenance of 
operating systems, application servers and middleware platforms is expensive and requires sufficient system administrators with the appropriate skills. This would make PaaS a more interesting choice for software vendors willing to enter the SaaS market. As with any paradigm shift, many new and somehow competing technologies enter the arena; more technologies emerge and compete when the new paradigm (SaaS in cloud computing in the context of this research) is promising major changes in the landscape of solution and platform providers. These circumstances generate an imminent need to investigate and conduct independent research on the relative value and performance of new types of technologies.

A comparable situation has emerged, for example in the eighties, when performance-critical software applications were expected to be executed by various kinds of parallel computing technologies, ranging from MIMD [13], SIMD [74], sharedmemory environments [75] etc. Very often, parallel computing architectures came along with specific programming paradigms and tools for parallel computing, such as parallelizing compilers [39], message-passing environments [76] etc. Back then, the research community had to deliver a lot of practical research to share experience and know-how, and to drive the qualitative improvement of the corresponding platforms. Today the domain of PaaS platforms that support the paradigm shift towards SaaS in cloud computing needs similar research.

The general research question that is addressed in this paper therefore is to which extent different types of Platform-as-a-Service solutions support the development and deployment of SaaS applications that start from an existing code base. The approach that has been applied is case study driven. The specific scope of the paper is in principle limited to the broad category of enterprise applications (such as the ones based on Java EE and .NET).

Clearly, PaaS offerings need a critical assessment: can PaaS platforms enable software developers to build and deliver SaaS applications without the complexity of dealing with the underpinning middleware and infrastructure? This experience report compares PaaS platforms with respect to their support of SaaS application development. We distinguish between the following three categories of PaaS platforms:

Category 1: PaaS platforms that mimic and match the APIs of popular enterprise application servers and middleware platforms. Examples are Windows Azure [46] using the .NET framework and the IIS web server, Red Hat OpenShift [63] based on the JBoss platform, Oracle Cloud [59] running on top of the WebLogic Server, and Cloud Foundry [80] using VMware and Spring technology.

Category 2: Focused PaaS platforms that aim to optimally support specific types of cloud applications. These platforms typically deploy other middleware and storage facilities that supposedly scale better. Google App Engine [32] and GigaSpaces' XAP Elastic Application Platform [31] belong to this category.

Category 3: Metadata-driven PaaS platforms. Similar to focused PaaS platforms, these platforms are designed with SaaS applications in mind. In addition, metadatadriven PaaS platforms introduce a higher-level composition and configuration interface that makes abstraction of the typical middleware level. However, this limits the complexity of the applications that can be implemented. Examples include Force.com [67,82], WOLF [83], and TCS InstantApps [71]. 
In order to compare these PaaS categories, we have reengineered an on-premise application towards the SaaS model. We have performed this task three times, each time yielding a working application on top of one representative platform for each of the categories listed above. In particular: we have used Windows Azure (category 1), Google App Engine (category 2), and Force.com (category 3).

We have addressed three requirements of SaaS providers with respect to support for SaaS application development. (1) The cloud platform should support the portability of SaaS applications [14,61], between the on-premise implementation and the SaaS-enabled implementations. Portability aims for minimizing the development and code migration efforts when re-engineering an application towards a PaaS deployment environment. (2) Furthermore, the platform should support the creation and management of multi-tenant applications $[19,36]$, which is critical to achieve economies of scale. (3) Finally, tool support is essential for SaaS developers to facilitate development and testing on top of PaaS platforms [30]. Other requirements, for instance regarding deployment, scalability and security, are out of scope of this paper. We have conducted this case study in a disciplined and systematic way. The outcome is explained in detail in this paper, and the practical and technical results are publicly available $^{1}$ to enable reproduction of the case study, or more likely, extension of the acquired experience and know-how by adding either application case studies or implementations on new and/or evolved PaaS platforms. We believe that there is a lack of in-depth case studies that reach beyond the exploratory use of PaaS platforms, thus yielding high-level impressions rather than in-depth experience.

The contributions of this work consist of (i) the elaboration of the requirements for the development of multi-tenant SaaS applications, (ii) the comparison of three different and representative PaaS platforms with respect to these requirements based on hands-on experience in a practical case study, (iii) the identification of research challenges for the improvement of current PaaS platforms in terms of supporting SaaS application development, and (iv) an understanding of the impact of the selection of (types of) PaaS platforms when developing SaaS applications.

The remainder of this paper is structured as follows. Section 2 elaborates on the PaaS requirements imposed by SaaS developers and presents the case study that is used throughout this paper. The representative PaaS platforms are introduced in Section 3. Section 4 compares the different PaaS platforms with respect to portability. Section 5 covers the support for multi-tenancy, and Section 6 addresses the tool support for SaaS developers. Section 7 discusses the results and identifies the challenges ahead. Section 8 covers related work. Section 9 concludes the paper.

\section{Requirements \& Illustration}

First we define the requirements that software vendors must consider when developing enterprise SaaS applications on top of PaaS platforms. Furthermore, we illustrate and further motivate these requirements by describing the application case study.

The focus of this paper is on two types of stakeholders: PaaS providers and SaaS providers. SaaS providers offer on-demand software services to their tenants, poten-

1 https://distrinet.cs.kuleuven.be/projects/CUSTOMSS/comp/ 
tially using a PaaS platform to develop and deploy these SaaS applications. The SaaS provider often becomes a customer of the PaaS provider. Another relevant stakeholder for this paper is the tenant administrator. Each tenant should assign this special role to someone who is responsible for managing the provided SaaS application on behalf of the tenant, for example to configure the tenant-specific preferences.

\subsection{Requirements}

The requirements that are central to this study are somehow trivial if one considers the overall context of the paradigm shift to $\mathrm{SaaS}$ in cloud computing: if software vendors consider evolving to become a SaaS provider, then they typically have economical concerns such as the cost of re-engineering the software to become SaaS-enabled, as well as the cost of delivering service based on the resulting ( $\mathrm{SaaS}$ ) solution ${ }^{2}$. As for the cost of re-engineering, this concern translates directly into the requirement of application portability and into a second requirement of tool support that can further reduce the efforts that are required to develop and deploy on a specific PaaS platform. As for the cost of delivering service, it is important that investments can be shared for a maximal set of clients; this makes multi-tenancy extremely important in this study.

This section therefore elaborates on three key requirements: (i) support for portability of SaaS applications, (ii) support for creating and managing multi-tenant-aware applications, and (iii) availability of tool support for SaaS developers (e.g. development and testing tools).

Portability. Software portability is the ability to easily deploy and use applications on different environments (e.g. operating systems and middleware platforms). In literature, a software application is defined as portable when the cost and effort to port and adapt it to a new environment is less than the cost of redevelopment of a new version and training of the developers [51,29]. Portability is a desirable property for SaaS applications $[14,61]$. From the perspective of the SaaS providers, portability enables them to port and adapt a SaaS application to different platforms (on premise as well as in the cloud) with minimal changes. This will subsequently enable the deployment and execution on multiple PaaS platforms of the same type, and even enable a hybrid approach (both external PaaS platform and in-house). In addition, the application easily works with other applications on local and remote systems, and developers require little or no retraining. The requirements on portability are decomposed into the following subrequirements:

1. Compatibility with mainstream programming models and middleware frameworks is necessary to ensure portability of the application code to several platforms, without requiring the rewriting of the SaaS application or extensive training of the SaaS developers. This includes all software concepts for software engineering activities throughout the software life cycle, such as programming models, component models, testing and deployment.

\footnotetext{
2 This is a generic observation, early practitioners of parallel computing shared similar and essential concerns.
} 
The SaaS applications are developed and deployed on top of a PaaS platform instead of the traditional middleware container used in the on-premise model (e.g. Java EE or .NET). Such a container provides a specific programming model and a set of common services (often based on standards), covering the nonfunctional requirements of the application, such as persistence and security. However, PaaS platforms also have to support these common programming concepts and middleware frameworks, without tight integration between application and platform, to facilitate the development and to enable portability.

2. A PaaS platform should offer support for easy integration with cloud services, both internal and external. Typically, a PaaS platform offers additional, cloudspecific services. For example, a cloud environment needs a highly performant and scalable datastore and distributed caching. Furthermore a service-oriented environment also requires composition with external (cloud) services and applications, such as scalable cloud storage. Therefore, the portability requirement applies to the platform-provided as well as external (cloud) services that are used by the SaaS application.

Multi-tenancy. As explained above, multi-tenancy is one of the key enablers for the SaaS model to achieve economies of scale and operational cost efficiency [19,81], but it also introduces some complexity [36]. Therefore a PaaS platform should offer SaaS providers a way to endow applications with multi-tenancy, in a transparent way or via an API, in order to allow them to benefit from the improved cost efficiency. To enable multi-tenancy at the application level the following subrequirements should be fulfilled [36,11]:

1. The core idea behind multi-tenancy is the sharing of both infrastructure and software among all tenants. Therefore data isolation between the different tenants is a key requirement that needs to be ensured, for instance by means of a multi-tenant database, to prevent that tenants can access data belonging to other tenants. In addition, the tenant of an incoming user request should be identified (via a tenant ID) and stored as long as the request is processed (i.e. the tenant context).

2. Each tenant has its specific needs. Therefore tenant-specific configuration and customization are critical to satisfy the different requirements [78]. However, these customizations should only be applied to a specific tenant and may not affect the other tenants. In addition, a configuration interface is required to enable the selection of tenant-specific customizations in a safe and consistent way. Each tenant should assign the tenant administrator role to someone who is responsible for configuring the SaaS application to the tenant-specific preferences, setting up the application data (e.g. user management), and monitoring the overall service.

3. In order to manage and control multi-tenant applications, SaaS providers need a tenant-specific application management facility. Key requirements with respect to application management are billing and metering. The SaaS provider should be able to monitor the SaaS application on a per tenant basis to determine the resource consumption by a particular tenant (for pay-per-use billing) and to enforce the different service level agreements (SLA). On the other hand, tenants want to monitor the total usage by their users and check whether the agreed SLAs are provided. 
Tool support. Tool support is crucial for the (fast) adoption and ease of use of new technologies. We identified the following subrequirements [30]:

1. A development environment is required to facilitate the development of SaaS applications using the programming model of a specific PaaS platform. Eclipse and Visual Studio are examples of popular IDEs among developers.

2. The developed SaaS application will be deployed on the PaaS platform. A local development server and/or storage offers SaaS developers the possibility to easily test the application on a local environment simulating the PaaS environment, and to optimize the deployment configuration. This includes support for different types of databases.

3. A testing framework is required to efficiently test applications, for instance by means of executing unit tests. These testing tools should be able to interact with the cloud platform (or the simulated environment) and other provided services.

\subsection{Case study}

This section presents the case study in the travel business domain that facilitates the analysis of the development support provided by the different PaaS platforms.

\subsubsection{Online hotel booking.}

A software vendor sells hotel booking systems to travel agencies. This allows the travel agencies to offer their customers an online service to search and book rooms in hotels. Typically, this hotel booking application is deployed on a local server or hosted by the software vendor. The latter is based on a traditional application service provisioning (ASP) model: each travel agency has its own, separate application instance (single-tenancy).

The software vendor decides to offer the online booking application to its customers as a multi-tenant SaaS application, customizable to the different requirements of the travel agencies. The base application is offered at a low cost, but additional services and features can be selected at an additional charge to fit the tenant-specific requirements. In this case the travel agencies play the role of tenant whereas the employees and customers of a travel agency are considered the end users. In addition, the travel agencies have to assign the tenant administrator role to someone of their ICT staff to specify the tenant-specific customization preferences, and to set up the application data (e.g. user management) via the configuration interface. In this example the configuration is limited to the selection of a price calculation implementation.

With the multi-tenant SaaS model all tenants share one or more instances of the online hotel booking application, instead of serving a separate application instance per tenant. This approach reduces the operational costs significantly for the software vendor: (i) hardware and software infrastructure can be more cost-efficiently divided among the tenants, and (ii) upgrades of the software only have to be applied to the shared instance(s) and all tenants will automatically use the most recent version. To provide a scalable platform that allows the travel agencies to cope with the seasonal 


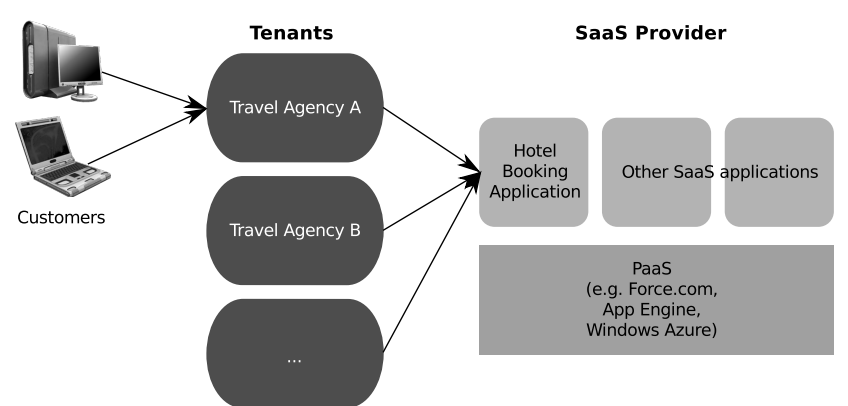

Fig. 1 The hotel booking application with SaaS deployment model

peeks in booking requests, the software provider wants to deploy the SaaS application on a PaaS platform, as depicted in Fig. 1. In principle, this deployment model has the additional advantage that the PaaS platform takes care of the infrastructure-related concerns, and the software vendor can focus on the development and maintenance of the hotel booking application.

The core design of the hotel booking application (Fig. 2) consists of two major parts: the data model and the business logic. The data model describes the main entities in the travel business domain. A Hotel consists of a name, an Address and a list of rooms. The information about a Room is captured in a RoomDetails object (e.g. the room number and the price per night). Each Room also contains a list of bookings. Booking contains the details of a booking: the name of the customer that makes the booking, a start and end date (booking period), the total price of the booking and the name of the hotel as well as the room number.

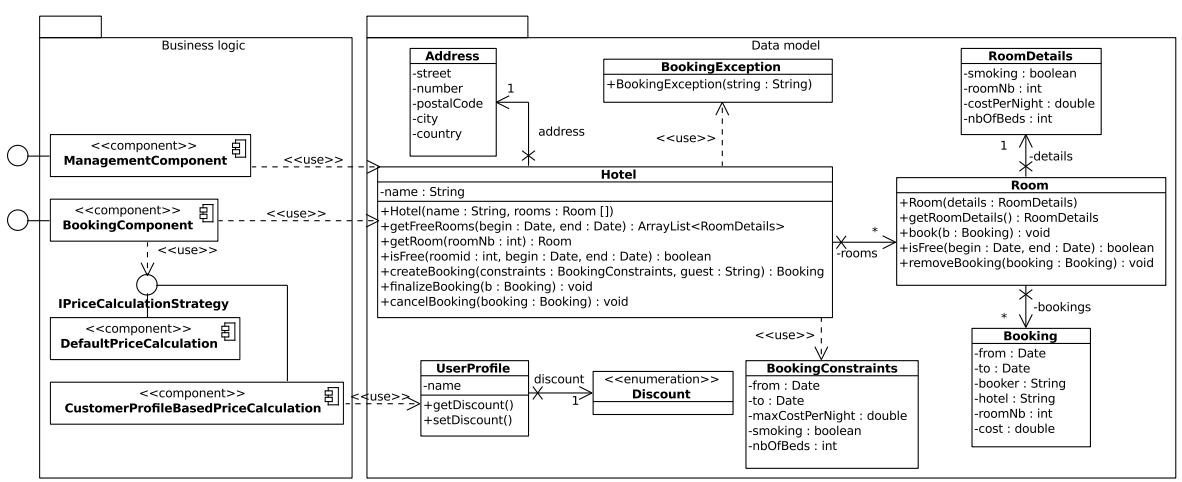

Fig. 2 Design of the hotel booking application

The business tier contains the different components to perform bookings of rooms (BookingComponent) and to manage the application data (ManagementComponent). These components also provide interfaces that can be used by a front end. The management interface enables the travel agencies to (un)register hotels and to get an overview of the bookings. 
Customers can make inquiries about the availability of rooms during a certain period (using getFreeRooms (Date, Date)) in the currently selected hotel. It also provides methods to book rooms: createBooking (BookingConstraints, Guest) creates a tentative booking which can be finalized by using finalizeBooking (Booking). It is possible to cancel a booking using cancelBooking(Booking). Customers can pass their requirements by means of BookingConstraints.

A customer has the opportunity to make several bookings in multiple hotels concurrently. Therefore, conversational state, such as the currently selected hotel and the list of tentative bookings, should be kept on the server for the duration of the customer's session. The finalizeBookings (List<Booking>) operation of the BookingComponent finalizes all tentative reservations in the customer's session.

When a new booking is created, the total price is calculated by IPriceCalculatorStrategy. In this simplified case study, price calculation is offered as a feature with two available implementations: (i) the default approach, i.e. multiplication of the number of nights and the price per night, and (ii) a discount is assigned to customers based on their profile (e.g. regular customers get a $10 \%$ discount on their bookings). In the multi-tenant SaaS application, the tenants have to select one of these feature implementations. Thus different tenants can have a different configuration.

\subsubsection{Implementation highlights.}

We have implemented an on-premise version of the hotel booking application as a component-based web application, using Java EE as well as the .NET Framework (see Table 1).

Table 1 Overview of the quantitative metrics of the size of the Java EE and .NET versions of the onpremise hotel booking application, expressed in lines of code (comments and empty lines not included). These metrics are used to evaluate the portability to the different PaaS platforms. Build and query scripts are not taken into account.

\begin{tabular}{c||c|c|c} 
& Type & \# files & \# lines of code \\
\hline \hline \multirow{4}{*}{ Java EE } & Java & 28 & 1210 \\
& JSP & 13 & 490 \\
& XML (config) & 4 & 106 \\
& HTML & 3 & 19 \\
\multirow{4}{*}{.NET } & CSS & 1 & 61 \\
& C \# & 37 & 1280 \\
& ASP.NET web page & 20 & 788 \\
& XML (ASP.NET config) & 6 & 118 \\
& CSS & 1 & 274
\end{tabular}

In the Java EE version we used Enterprise JavaBeans (EJB) [25], a server-side component architecture, for the business logic and the Java Persistence API (JPA) [24], a standard framework for managing relational data, for the data model. JPA also defines an object-oriented query language for entities stored in a relational database, called the Java Persistence Query Language (JPQL). Further, we developed a web tier as front end using Java Servlet [52] technology and Java Server Pages (JSP). 
The .NET version is implemented using the ASP.NET MVC 3 framework [45], a web application framework that implements the Model-View-Controller (MVC) pattern. For persistence we used the ADO.NET Entity Framework [44], i.e. a set of data-access APIs similar to JPA. It is commonly used by programmers to access and modify data stored in relational database systems, though it can also access data in non-relational sources. Data querying capabilities are provided via the .NET Language Integrated Query (LINQ) framework. The business logic is implemented in basic C\#.

For the on-premise Java EE version we used the Eclipse development environment, the Glassfish application server (with TopLink SQL database), and JUnit as testing framework. The tool support for the .NET implementation consisted of Visual Studio 2010 (with Unit Testing Framework), the Internet Information Services (IIS) web server, and SQL Server Express.

\subsection{Approach}

The case study has been conducted in a systematic way by starting from a stable and operational implementation of the proposed enterprise application, offering a well-specified functionality as summarized above. For all the platforms that have been addressed, we have conducted the minimal but sufficient modifications to deliver exactly the same functionality of the corresponding enterprise application. We have reported systematically on our activities and we then had two independent scores by experts to express the quality of the underpinning PaaS environment.

First, we have migrated the on-premise application to a SaaS application on top of the different PaaS platforms, without taking into account multi-tenancy. After this first step, quantitative results with respect to portability have been gathered in the form of the amount of lines of code that have been changed compared to the onpremise version. This results into a score for the compatibility requirement. Furthermore, scores are assigned to each platform to express the quality of the provided integration support.

Next, the SaaS implementations on the different platforms have been adapted and extended (if necessary) to support application-level multi-tenancy. Subsequently, a qualitative score is assigned for each subrequirement of the multi-tenancy support (as described in Section 2.1). This score is mainly based on the availability of built-in support to address the specific requirement and to which degree. When a platform does not provide built-in support, we have validated whether it is possible to fulfill the requirement by means of a custom implementation, and we have assessed how much effort such custom implementation requires (in terms of lines of code).

Finally, the quality of the tool support associated with each platform has been assessed based on the hands-on experience with these tools during the entire development process. Again, scores have been generated for each individual subrequirement.

The details of the different case study implementations and results are discussed for each requirement separately in Sections 4, 5, and 6. In addition, with each new release of a platform that has been investigated, the implementations and scores have been updated (when necessary). These updates were logged to keep track of the evo- 
lution of the platforms. Relevant findings with respect to these new releases are discussed in Section 7.

\section{Presentation of the PaaS Platforms}

We distinguished three categories to classify PaaS platforms with respect to their support of SaaS application development (Table 2). For each category we selected a mature and representative platform to compare these different categories, in particular Force.com [67,82] (Category 3), Google App Engine [32] (Category 2), and Windows Azure [46] (Category 1). This section summarizes each of the PaaS platform that have been selected. Furthermore, the specific setup and version that we used during the comparison are specified.

Table 2 Classification of the major PaaS platforms into the three categories we distinguished. The majority of the current PaaS platforms belong to category 1, because they build on common application servers and middleware platforms and focus on migration of existing applications to the cloud.

\begin{tabular}{c||cc}
\multicolumn{1}{c||}{} & \multicolumn{2}{c}{ PaaS platforms } \\
\hline \hline & Amazon Elastic Beanstalk [3] & Oracle PaaS [59] \\
Category 1 & Cloud Foundry [80] & Red Hat OpenShift [63] \\
& CloudBees [22] & SAP NetWeaver [69] \\
& Heroku [37] & Windows Azure [46] \\
& IBM SmartCloud [40] & WSO2 Stratos [84,9] \\
\hline \multirow{2}{*}{ Category 2 } & AppScale [18] & Google App Engine [32] \\
& GigaSpaces' XAP Elastic Application Platform [31] \\
\hline \multirow{2}{*}{ Category 3 } & Cordys PaaS [23] & TCS InstantApps [71] \\
& Force.com [67,82] & WOLF [83]
\end{tabular}

3.1 Force.com (Version 19.0 - 25.0) - Category 3

Force.com $[67,82]$ is a cloud computing platform that makes the core technologies behind Salesforce CRM [68] available for developing and deploying custom enterprise applications, and also extensions for the Salesforce CRM application. It provides a development environment to deliver business applications that run against the Salesforce.com database (data-centric) [6]. The targeted consumers are private enterprises and commercial software providers.

The key enabling technologies of the Force.com platform are multi-tenancy and metadata. Everything exposed to developers and application users is stored internally as metadata. At runtime the required application components are generated by the runtime engine (kernel), based on these metadata [82]. These application components are combined into a polymorphic application, fulfilling the individual expectations of various tenants and their users [82]. As depicted in Fig. 3, the underpinning database clearly separates the metadata describing the base functionality of an application, the metadata corresponding to tenant-specific customizations, and the actual application data. Custom business logic and functionality can be written in Apex, a strongly 


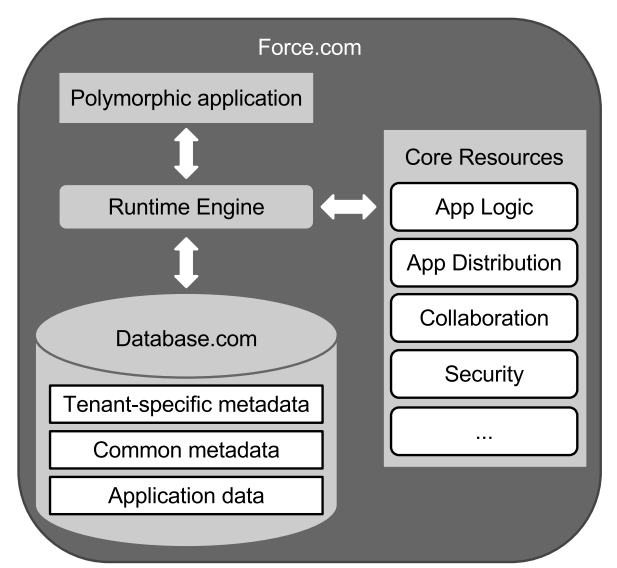

Fig. 3 Force.com's metadata-driven architecture, based on [82]

typed, object-oriented programming language (part of App Logic in Fig. 3). Other cloud services provided by the Force.com platform, called core resources, are for instance AppExchange (App Distribution), a marketplace for cloud applications, and Chatter (Collaboration), a suite of collaboration and social features.

As is typical for PaaS platforms of category 3, Force.com applies a different approach and programming model compared to the traditonal middleware frameworks because of the metadata-driven architecture. It takes a more scripting-based approach using a dynamically interpreted language (i.e. Apex). We used the pure Force.com platform without third-party extensions for implementing the application case. The implementation is developed on versions 19.0 (Summer '10) till 25.0 (Summer '12) of the Force.com platform.

\subsection{Google App Engine (Java SDK 1.3.6 - 1.6.0) - Category 2}

Google App Engine (GAE) [32] is a PaaS plaform that enables the development and hosting of web applications on the same systems that power Google applications. It is targeted at traditional web applications, but it is less suitable for general-purpose computing [6]. This allows Google to enforce a clean separation between a stateless computation tier and a stateful storage tier. Furthermore, GAE supports automatic scaling and load balancing as the needs for traffic and data storage grow. To handle large and complex requests or to do continuous processing, GAE provides more powerful backend instances. Backends do not automatically scale based on the load and can retain the state of the application.

The following programming languages are supported:

- Standard Java technologies, including the JVM, Java servlets and the Java programming language, or any other language using a JVM-based interpreter or compiler (e.g. JavaScript, Ruby and Scala).

- The Python progamming language, including the Python standard library. 
The Java and Python runtime environments are implemented as a secure sandbox where an application can run without interference from other applications, independent from the underlying hardware and operating system. In the rest of this paper the discussion is limited to the GAE's Java runtime environment.

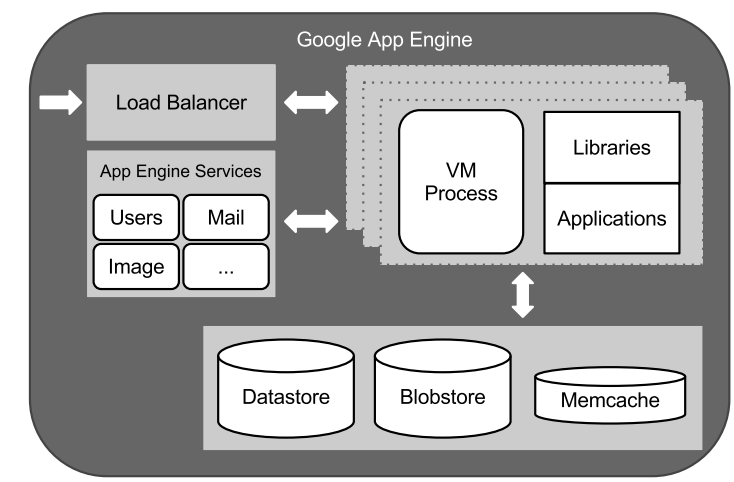

Fig. 4 Overview of the Google App Engine architecture

A simplified overview of the GAE architecture is presented in Fig. 4. Incoming requests are routed to an application server by a load balancer. Clones of the application are automatically created when needed. The platform provides a variety of cloud services, such as the users and mail services, to support application development. At the lowest level a distributed datastore service provides schemaless object storage, a blobstore serves binary large objects (called blobs), and a memcache service offers a high performance in-memory key-value cache. The datastore consists of the MegaStore storage system [10], an abstraction layer built on top of BigTable [15]. It combines the scalability of a NoSQL datastore with the convenience of a traditional relational database. The datastore is divided into separately replicated partitions, with full ACID semantics within these partitions, but limited consistency guarantees across them [10]. In addition, only the scalable traditional relational database features are supported.

For this comparison we used GAE with Guice [33], Google's dependency injection framework, to add better modularity and customization capabilities to the platform. This allows us to develop the price calculation variation as a module that can dynamically be selected when needed. We used GAE SDKs 1.3.6 till 1.6.0 in this comparison.

\subsection{Windows Azure (SDK 1.2 - 1.6, C\#) - Category 1}

The Windows Azure platform [46] is a cloud computing platform supporting the development and deployment of Windows applications that provide services to both businesses and consumers. These applications can be created using the .NET framework in languages such as $\mathrm{C \#}$ and Visual Basic, or they can be built without the 
.NET framework in C++, Java, PHP, Ruby and Python. Windows Azure is originally a PaaS platform but since November 2010 it is also possible to deploy virtual machine (VM) images of Windows Server (2008), which is a form of IaaS. Notice that Windows Azure provides not necessarily an execution environment for all supported languages. For example, Java APIs are available for the different Azure services, but the developer has to install a JVM and an application server in an Azure VM himself.

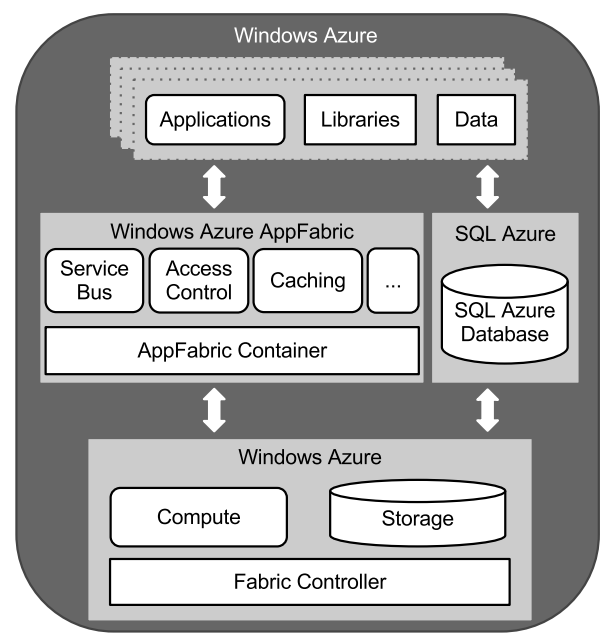

Fig. 5 Overview of the Windows Azure architecture [16]

The Windows Azure platform is a group of cloud technologies, each providing a specific set of services to application developers [16] (see Fig. 5):

- Windows Azure: A Windows environment for running applications (Compute) and storing data (Storage) in the cloud. A Windows Azure application can be created using three kinds of compute service types, called roles: (i) a Web role for running web-based applications, (ii) a Worker role for (background) processing, and (iii) a VM role to run VM images. An application is structured as any combination of role instances. Web and worker role instances consist of a Windows Server 2008 R2 VM with the .NET 4.0 framework pre-installed. In a web role, there is also an IIS web server available.

The storage service consists of blob storage (course-grained, unstructured data), table storage (set of entities with properties, NoSQL), and queues (asynchronous communication between different roles). All data is accessible via a REST API and the OData query language for tables. The Fabric Controller makes abstraction of the underlying infrastructure and offers a pool of processing power to the compute and storage services.

- SQL Azure: A cloud-based service for relational data, built on Microsoft SQL Server. 
- Windows Azure AppFabric: A middleware platform on top of Windows Azure that provides a higher level of abstraction and reduces the complexity of cloud development [47]. The AppFabric Container provides a new programming model and runtime for cloud application development using .NET core languages [53]. The AppFabric Container itself is not publicly available and therefore not further discussed in this paper, but Microsoft offers several AppFabric Services on top of this container, for example caching. Recently, the AppFabric Services are renamed to Windows Azure Features.

The Windows Azure platform provides an environment that strongly corresponds to a traditional on-premise setup (cf. Category 1), this especially applies to the Windows Azure Compute service and SQL Azure. In this comparison we focus on Windows Azure using traditional .NET frameworks in the C\# programming language and the Ninject dependency injection framework [42]. We also include SQL Azure into the comparison. The case study application is implemented using Azure SDKs 1.2 - 1.6.

\section{Portability}

The main driver behind portability over PaaS platforms is the need (by SaaS providers) for support to facilitate the transition from on-premise towards the SaaS-based deployment models. More specifically, we have identified the following subrequirements: (i) compatibility with the mainstream programming model and middleware frameworks, and (ii) easy integration with cloud services, platform-specific as well as external.

The comparison of the portability support is structured as follows. We describe for each platform first the compatibility of the programming model and then the integration support. The former subrequirement discusses a) the platform-provided programming model and middleware, and b) the extensibility of the platform. The latter consists of integration with platform-specific services, and external (cloud) services and applications. Finally we give a brief summary of our findings regarding portability. Table 3 presents an overview of the portability support on the three PaaS platforms.

\subsection{Force.com}

The programming model of the Force.com platform is incompatible with the onpremise model of the hotel booking application. However, it provides extensive integration facilities with external applications and services on different (cloud) platforms and using other programming languages.

\subsubsection{Compatibility with mainstream technology.}

The metadata-driven architecture and the Salesforce.com-specific Apex language are incompatible with common programming models and middleware frameworks and therefore hinder portability across different platforms. Concretely, this means none of 
Table 3 Overview of portability support on the three investigated PaaS platforms.

\begin{tabular}{|c|c|c|}
\hline & Compatibility & Integration support \\
\hline Force.com & $\begin{array}{l}\text { Not compatible: } \\
\text { - Metadata-driven (e.g. data model) } \\
\text { - Apex language (Force.com-specific) }\end{array}$ & $\begin{array}{l}\text { - Apex integration with core resources } \\
\text { (e.g. database) } \\
\text { - Web Services \& REST API } \\
\text { - Including utilities (e.g. APIs) for } \\
\text { different programming languages } \\
\text { and platforms }\end{array}$ \\
\hline GAE & $\begin{array}{l}\text { Limited compatible: } \\
\text { - White list of Java classes (e.g. no EJB } \\
\text { or JAX-WS servers) } \\
\text { - JPA: limited + GAE-specific code } \\
\text { - Extensible: REST, Guice... (support } \\
\text { limited by white list) }\end{array}$ & $\begin{array}{l}\text { - Datastore (BigTable) via Megastore } \\
\text { - Google Apps services } \\
\text { - URL Fetch (low-level) } \\
\text { - Remote API (GAE-specific) }\end{array}$ \\
\hline Azure & $\begin{array}{l}\text { Fully compatible: } \\
\text { - Common .NET frameworks (MVC, } \\
\text { WCF, Unity...) } \\
\text { - Extensible by importing libraries } \\
\text { - ADO.NET EF: only SQL Azure }\end{array}$ & $\begin{array}{l}\text { - Platform-provided services via com- } \\
\text { mon .NET frameworks } \\
\text { - Azure Table Storage: no support } \\
\text { - Web Services \& REST API } \\
\text { - Service Bus (AppFabric) }\end{array}$ \\
\hline
\end{tabular}

the implementation artifacts of the on-premise versions can be reused. A full rewrite of the application is required. Due to this lack of compatibility with traditional programming models, it is also not possible to (re)use existing middleware frameworks on top of the Force.com platform. Such middleware support should be provided by the platform itself, or be specifically written for Force.com and made (publicly) available, for instance via the AppExchange marketplace. First, we describe the programming model used by Force.com. Next, the Apex language is introduced and illustrated.

Business objects. Force.com focuses on building data-centric business applications and this influences the way the application is designed. The developer has to reason in terms of business objects (BOs). Business objects represent the data that will be stored in the database. In contrast to for example entities in Java EE, BOs cannot contain any behavior. Moreover, they are not specified in a programming language (code), but have to be created via a point-and-click web interface and are stored as metadata in XML (Listing 1).

Relationships [17] between business objects are created by specifying relationship fields that map records in one object to records in another. Force.com has two types of relationship fields:

- The Lookup Relationship is used to create one-to-one and one-to-many relationships between objects, without a direct dependency.

- The Master-Detail Relationship creates a tight parent-child relationship between two objects. The relationship field is required on all child records and deletes are cascaded from the parent to the child. This relationship type also enables the creation of many-to-many relationships through the use of a junction object. 
Listing 1 A fragment of the raw metadata of a simple business object (BO) representing the address of a hotel. In total it consists of 100 lines of XML. Clearly, implementing even a simple BO is not recommended using this metadata representation.

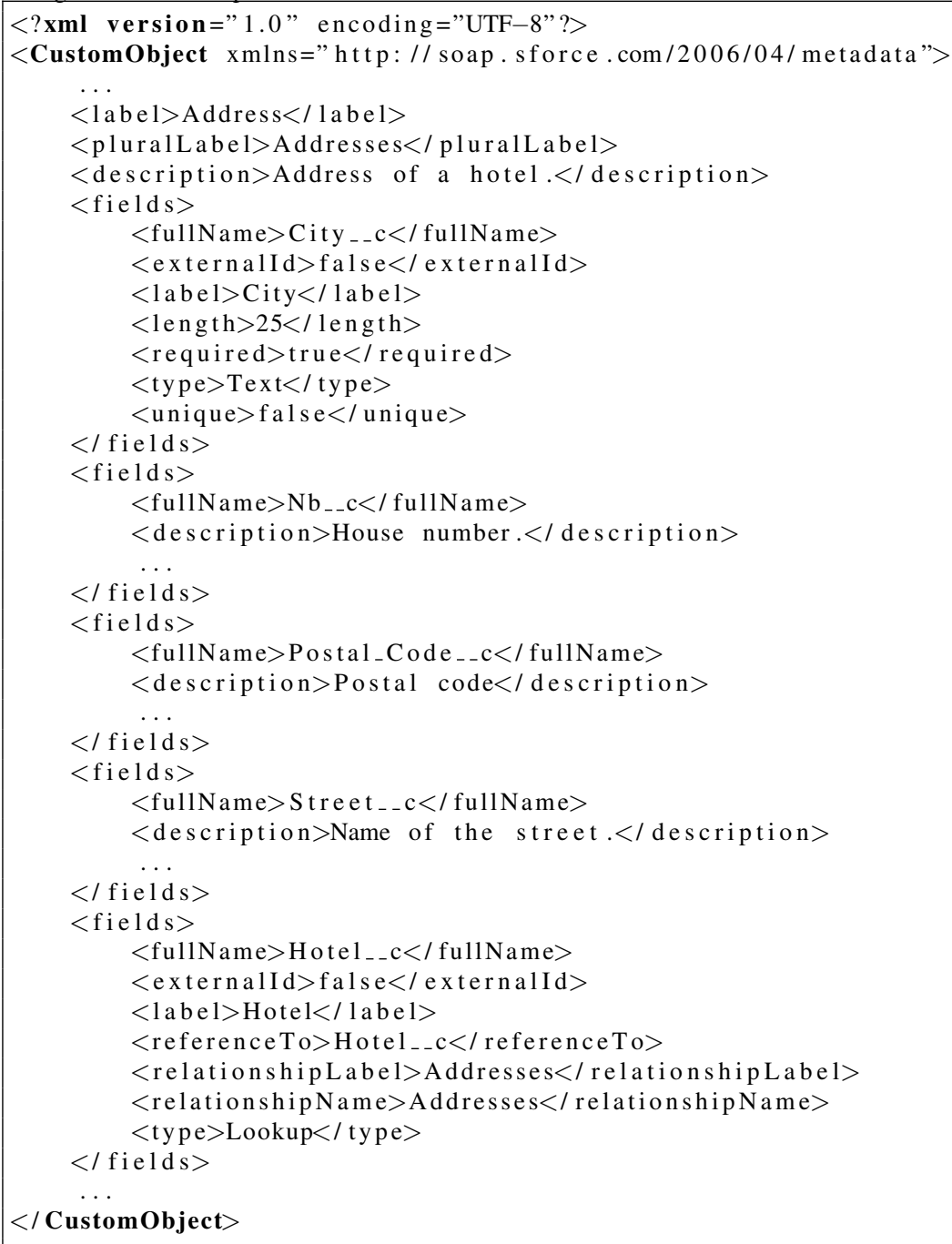

In the case study implementation on top of Force.com we used both relationship types. When a travel agency removes a hotel from the online hotel booking application, all rooms and bookings related to that hotel should also be deleted. Therefore the application needs master-detail relationships: rooms are details of a hotel, while bookings are details of room. However, the master-detail relationship requires that all relationship fields are set at the moment of object creation. This prevents creating tentative bookings that are not yet added to a room. To solve this issue, we had 
to extend our data model with an Invoice that manages all tentative bookings of a specific customer during one session (via a master-detail relationship), and stores the total price of these bookings in the invoice object. An invoice also has a status field, and only when the status of the invoice changes to 'closed' a booking is considered finalised. Between a Hotel and an Address we used a lookup relationship, as shown on lines 31-39 in Listing 1.

Apex. The Apex language is used to create, persist and update instantiations of business objects, and to query the database via a built-in query language. When a business object is materialized in the Apex language, it is called an sObject. sObject is also the generic abstract type, comparable with Object in Java. Furthermore the Apex language is used to implement controller classes for the user interface, to invoke external web services, and to expose operations in a web service.

Queries on the Force.com database are expressed in the Salesforce Object Query Language (SOQL). SOQL is an object query language (cf. JPQL for Java Persistence) that is integrated in the Apex language and uses the abstraction provided by the business objects and their relationships to navigate through the application data. The syntax is similar to the one of SQL, but it does not support all advanced features such as joins, wildcards or calculation expressions. Besides SOQL, Force.com also supports the Salesforce Object Search Language (SOSL) which enables text searches across all persisted objects.

Since a business object itself cannot validate input data, validation rules need to be specified separately. The validation rules consist of mathematical formulas that allow to compare input data with other data (predefined thresholds or data from other BOs). For instance, a validation rule checks whether the end date of a booking period is after the start date. However, these mathematical formulas are not powerful enough to perform all necessary validation checks. For example, it is not possible to define a loop that iterates over multiple BOs (in a list). This is required in the case study to check the availability of a room (by iterating over the different bookings of the room): a room may only be booked when there is no other booking in the same time period.

To implement more complex business logic and validation rules like checking the availability of a room, the Apex language needs to be used. By means of database triggers, pieces of Apex code are executed before or after events occur on an sObject, for instance the insertion of a BO. Apex triggers enable developers to change BOs when such events occur, or to perform complex validation checks.

For example, CheckBookingPeriod (Listing 2) is an Apex trigger in our case study implementation to check the availability of a room. For each newly inserted or updated booking, this trigger first retrieves via a SOQL query all finalized bookings for the same room as the triggered booking (lines 4-7). Next, the trigger checks whether there is an overlap between the booking period of the triggered booking and the finalized bookings (lines 10-18). An overlap will result in an error (lines 15-16). When no overlap is detected, the execution of the normal insert (or update) operation will continue (line 13). 
Listing 2 Apex trigger to check the booking period of a new Booking. It illustrates the use of the Apex language and other aspects of the Force.com programming model. Custom sObjects are represented by their sObject name with '-_c' appended to the end as a suffix, for instance Booking_-c. Relationship names in SOQL queries consist of the related object name followed by '__r', e.g. Room_r.

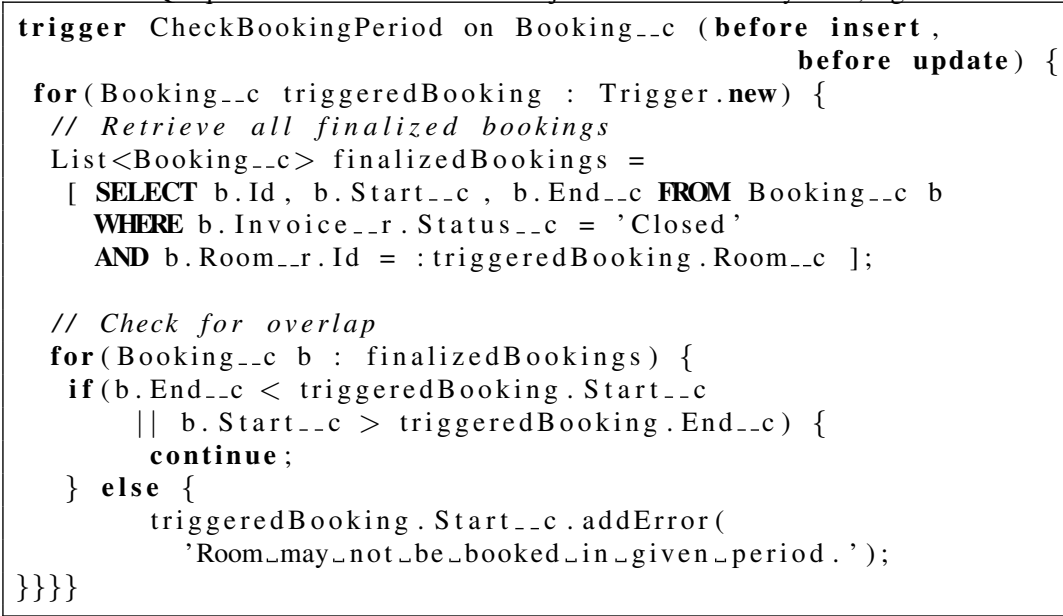

\subsubsection{Integration.}

The Force.com platform provides extensive integration capabilities. First of all, it offers a tight integration between the Apex language and the core resources (platformprovided services), for example the database. Apex is used for creating, modifying and querying database objects (via SOQL) and to write database triggers. Furthermore the language can be directly applied to implement user interface controllers, unit tests, and web services. Thanks to this integration with Apex, developers can use these facilities out of the box without having to write additional (integration) code.

Next, the platform provides support for integration with external applications and services that use other programming languages and platforms. Developers can create and expose SOAP-based web services using Apex, or invoke external SOAP- and REST-based web services from the platform. In addition, the Force.com Web Services and REST API provide direct access to the Force.com data from an external system. These APIs are fundamental integration points, allowing access via clients on any platform. To ease the integration with these external (client) applications, software utilities (called toolkits) are built around these core APIs. There exist toolkits for (i) programming languages such as Java, .NET and Ruby, (ii) cloud platforms such as Amazon Web Services [2] (S3 and EC2), Google App Engine [32] and Windows Azure [46], and (iii) mobile platforms like Android and iOS. Concretely, they support native development approaches with using the Force.com Web Services and REST APIs. Furthermore the Force.com database is also available as a standalone service (Database.com) using the same toolkits. This enhances the reusability of applications, data and services outside the Force.com environment. For example, a new 
(version of a) SaaS application can be developed on a different platform without having to port all data to the other platform.

Finally, additional integration facilities are available in the AppExchange marketplace, for instance ERP connectors and data integration solutions.

\subsection{Google App Engine}

Google App Engine (GAE) supports Java and other JVM-related languages and is extensible with common middleware frameworks. But this compatibility has its restrictions, limiting the portability across different Java-based platforms. Furthermore, the platform-provided cloud services are well supported. Integration with external services, however, is limited.

\subsubsection{Compatibility with mainstream technology.}

In contrast to Force.com, GAE is compatible with the common programming model used in the on-premise case study implementation by supporting Java and other JVMrelated languages, which facilitates the portability enormously. However, this compatibility is limited: to maintain scalability a number of restrictions are imposed, and some technologies and APIs are completely or partially unsupported by the underpinning platform. This results in adaptations to the application specifically for GAE.

In addition, GAE can be extended with other middleware frameworks, for instance Restlet [54] for RESTful web services. To use these frameworks additional (external) libraries have to be imported into the application. However, the imposed restrictions and incompatibilities also limit this extensibility. The result is that many middleware extensions do not work on top of GAE or require changes to the source code, which hinders an easy migration. For example, Restlet offers a separate edition, specifically adapted to fully work with GAE.

Major parts of the data and web tier of the Java EE version could be reused (Table 4). However, the business tier consisting of EJBs is not supported by GAE and therefore this logic is integrated into the web tier. The other code changes in the data and web tier are related to persistence. The different restrictions and incompatibilities imposed by GAE as well as the differences with respect to persistence are discussed in further detail in the following paragraphs.

Table 4 Reuse of code (in terms of lines of code) between Java EE version and GAE implementation. The last column indicates the ratio of the reusable code compared to the full GAE implementation.

\begin{tabular}{c||c|c|c|c} 
& \# common files & \# common LoC & \% reuse of Java EE & \% LoC of GAE \\
\hline \hline Data tier & 11 & 555 & $91.74 \%$ & $91.43 \%$ \\
Business tier & 0 & 0 & $0.00 \%$ & $/$ \\
Web tier & 28 & 916 & $90.42 \%$ & $76.21 \%$ \\
\hline Total & $\mathbf{3 9}$ & $\mathbf{1 4 7 1}$ & $\mathbf{7 8 . 0 0 \%}$ & $\mathbf{8 1 . 3 2 \%}$
\end{tabular}


Restrictions. A white list defines which Java classes of the JRE are allowed to be used on the platform [34]. For example, the APIs to write to the filesystem or to access the network are not available. Applications should use the provided datastore and URL Fetch services. Also the use of threads or other kinds of system calls are not allowed. Moreover web requests should be handled (in a single process) within 60 seconds. Processes that take longer to respond are terminated to avoid overloading of the web server [32]. Actually, GAE is optimized for applications with short-lived requests that take a few hundred milliseconds. These restrictions limit the options of the developer, but are not insurmountable: the restrictions and their alternatives need to be taken into account during application design. For example, the execution of long-running processes can be moved to backend GAE instances.

Unsupported. GAE offers built-in support for only a limited set of technologies. Among other things, this is due to the imposed restrictions. The web page "Will it play in Java?" [35] lists the level of compatibility with various Java technologies and frameworks. GAE's main focus is on web applications using Java Servlets and Java Server Pages (JSP), which have not a developer-friendly API to develop against. Enterprise Java Beans (EJB) and JAX-WS (SOAP Web Services) for servers are examples of two common technologies for enterprise applications that are not supported. Other technologies are only partially supported, for example JPA, the persistence API we used in the Java EE version of the online booking application.

Persistence. As explained in Section 3, GAE's datastore is based on BigTable [15], a NoSQL database, while JPA is a standard interface for interacting with relational databases. To support JPA the Megastore abstraction layer [10] provides an objectrelational mapping from RDBMS to NoSQL, though some features are not supported by this mapping, such as 'Join'-queries and aggregation queries. Therefore it is not possible to perform JPQL queries that use those more advanced features. An alternative for JPA is the Java Data Objects (JDO) persistence interface. JDO is independent of the underlying database and therefore a better choice when building a new GAE application.

However, independently from the used persistence interface, problems remain with the use of primary keys for entities. In common practice a primary key is a generated Long integer. Google App Engine, however, requires that in an entity relationship the children objects have a primary key that represents the parent object. Types that support such primary keys are a Key class, or a Key value encoded as a string. This Key class is a Google-specific class, which hinders portability. Listing 3 and 4 show a small entity representing the address of a hotel (child-parent relationship), respectively using default JPA and the version in GAE. Because the primary key refers to the parent object (i.e. the hotel), each child object can have only one parent. This limits the use of relationships and requires the data model to be structured in an hierarchical way (e.g. bidirectional bindings are not allowed).

Finally, it is not possible to use the ePersistenceContext annotation to declare a dependency to an entity manager, which is used to create, remove and query persistent entity instances. The developer has to manually create an instance of the 

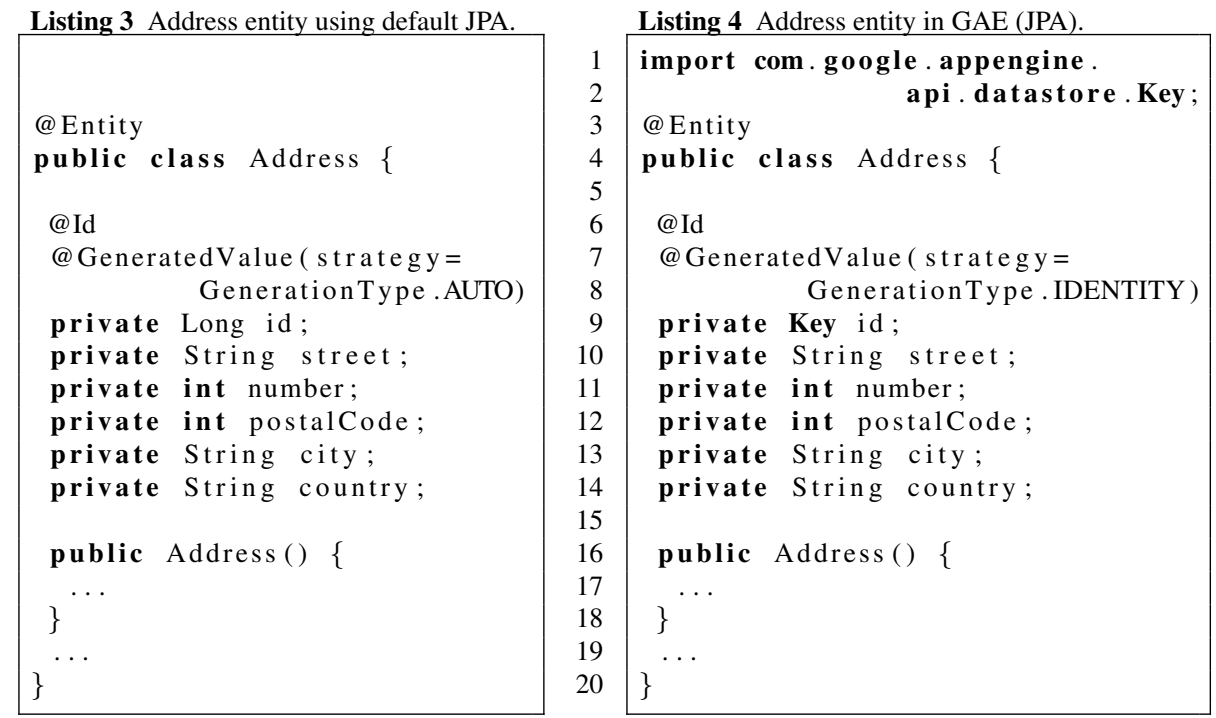

EntityManager when needed and close it afterwards, instead of it being managed by the underpinning middleware.

\subsubsection{Integration.}

While Force.com offers extensive integration support for platform-provided as well as external (cloud) services, Google App Engine clearly focuses on the integration of its own cloud services. The main platform-provided cloud services in GAE are the storage and caching services. Each of these services have an API that supports the integration with the application, often based on a standard, for example JPA, JDO, and JCache, a proposed interface standard for memory caches. The Megastore abstraction layer [10] provides the integration between the BigTable datastore [15] and the persistence interfaces by means of an object-relational mapping, though with some incompatibilities. Furthermore, several Google Apps services are well integrated into Google App Engine, for example Google Accounts for authentication via the User service API and Gmail to send emails via the Mail service API.

Built-in support for integration with external applications or resources, however, is limited. GAE applications can use the URL Fetch service, allowing $\operatorname{HTTP}(\mathrm{S})$ calls to other services (via low-level connections and streams). In addition, SOAP-based web services can be accessed by implementing a client using JAX-WS. Except for the Java Servlet API, GAE does not offer built-in support to make GAE applications accessible from external services and applications. To provide or call a RESTful web service, the SaaS provider has to import additional libraries, for example Jersey [58] and Restlet [54]. The latter even requires a GAE-specific library with limited features. Further, to develop a SOAP-based web service on top of GAE, SaaS developers have to directly use javax.xml and JAX-B, and implement their own handlers for SOAP 
messages. Finally, there exists a remote API to access the datastore of a GAE application remotely, but this requires the installation of the GAE SDK and it offers no standardized interface.

\subsection{Windows Azure}

Windows Azure is fully compatible with the existing .NET programming model and frameworks. In addition, many options are available for integration with external services and local as well as cloud applications. However, extensive support for integration with the Azure Table Storage is lacking.

\subsubsection{Compatibility with mainstream technology.}

In contrast to the white list of available classes in GAE, Windows Azure provides a full .NET stack, directly supporting commonly used .NET frameworks, such as the WCF framework [48] and ASP.NET MVC [45]. Thus, the programming model used for the .NET version of the case study implementation can be reused on Windows Azure without any compatibility issues, except for persistence in combination with Azure Table Storage. Also no restrictions like writing to the file system are imposed, although this should be done with caution in a cloud environment.

Furthermore, the platform can be extended with any third-party .NET framework, as long as this can be achieved by importing libraries. If an installation is required to extend the platform, then a VM role should be used, because installing is not allowed in the web and worker roles. However, in this case the SaaS provider will have to manage and maintain the whole stack himself.

When using SQL Azure, 100\% of the on-premise .NET implementation could be reused (Table 5). Only some additional configuration files were required to use the application on top of Azure. In the case that Azure Table Storage is used, less code of the on-premise application can be reused. The majority of changes are related to the data tier, where a custom (and probably not reusable) persistence layer had to be developed to integrate with the Azure Table Storage. This issue with the Azure Table Storage is explained in depth below.

Persistence. For persistence the SaaS provider has the choice between the Azure cloud storage (providing blobs, tables and queues), or SQL Azure (a relational database deployed in the cloud). The latter option supports a transparent migration: applications can access data stored in SQL Azure using the ADO.NET Entity Framework, in the same way as accessing SQL Server locally. The major difference with the local version is that the SQL Azure database is located outside the SaaS provider's boundaries. Therefore we have to change the connection string. In Listing 5 the implementation of an Address entity is presented. While in Force.com validation rules for BOs are separately defined, the ADO.NET Entity Framework allows developers to add validation rules as annotations.

On the other hand, the Azure cloud storage provides a schemaless persistence service with a much higher scalability than SQL Azure (comparable with Google's 
Table 5 Reuse of code (in lines of code) between .NET version and Azure implementations. The last column indicates the ratio of the reusable code compared to the full Azure implementations.

\begin{tabular}{c||c|c|c|c} 
& \# common files & \# common LoC & \% reuse of .NET & \% LoC of Azure \\
\hline \hline SQL Azure & & & & \\
Data tier & 23 & 584 & $100.00 \%$ & $100.00 \%$ \\
Business tier & 8 & 292 & $100.00 \%$ & $100.00 \%$ \\
Web tier & 33 & 1584 & $100.00 \%$ & $94.45 \%$ \\
\hline Total & $\mathbf{6 4}$ & $\mathbf{2 4 6 0}$ & $\mathbf{1 0 0 . 0 0 \%}$ & $\mathbf{9 6 . 3 6 \%}$ \\
& & & & \\
\hline \hline Azure Table & & & & \\
Data tier & 21 & 512 & $87.67 \%$ & $47.50 \%$ \\
Business tier & 8 & 247 & $84.59 \%$ & $76.00 \%$ \\
Web tier & 33 & 1572 & $99.24 \%$ & $93.96 \%$ \\
\hline Total & $\mathbf{6 2}$ & $\mathbf{2 3 3 1}$ & $\mathbf{9 7 . 7 6 \%}$ & $\mathbf{7 5 . 7 8 \%}$
\end{tabular}

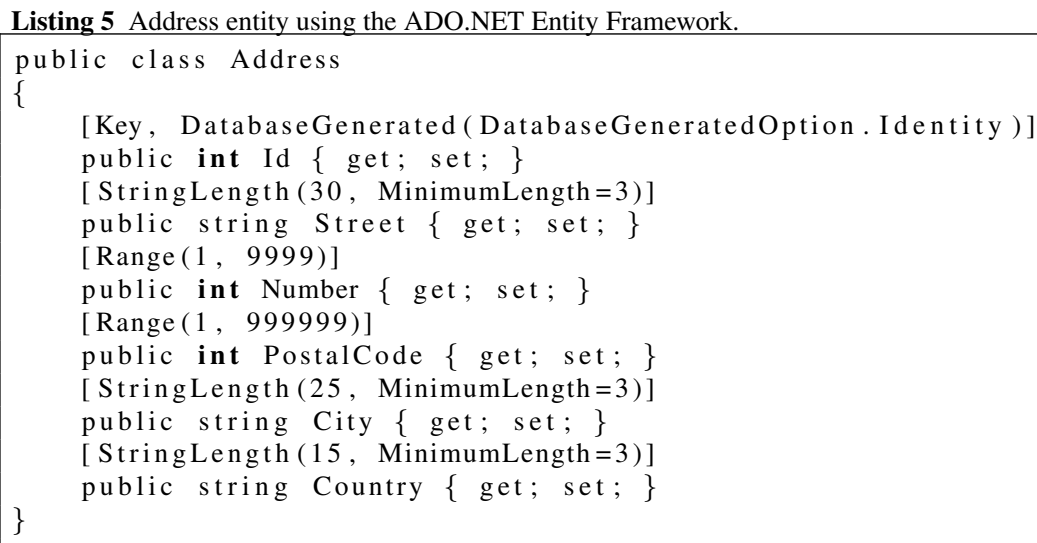

BigTable). As Google App Engine does inherently, the Azure Table Storage can be used as an alternative for SQL Azure. However, the ADO.NET Entity Framework (EF) is not compatible with the Azure Table Storage, because it is an object-relational mapping (ORM) framework. Therefore, there exists no programming support for domain entities and entity relationships when using the Azure Table Storage. In our implementation we decided to represent an Address entity the same way as in Listing 5, but without a (generated) identifier (without lines 3-4). The validation rules can be used by the MVC framework to do input validation.

\subsubsection{Integration.}

Windows Azure offers extensive integration facilities for platform-provided services as well as external services and applications. The main platform-provided services are the storage services and the AppFabric services (i.e. service bus, access control and caching) [47]. The programming model for these services is often based on common .NET frameworks, such as the ADO.NET Entity Framework (for SQL Azure) 
and WCF [48], which enables easy integration. The caching service provides an API that is comparable to the Memcache service of GAE, and can be used after limited configuration changes. However, the Azure Table Storage is not well integrated in the programming model. Integration with external services and applications is provisioned by the Service Bus by AppFrabric and the use of SOAP- and REST-based web services. In the following paragraphs we discuss in depth the issues with the Azure Table Storage, and integration with external services and applications.

Azure Table Storage. In GAE, the Megastore abstraction layer hides the complexity of the BigTable datastore by providing a mapping from RDBMS (JPA) to NoSQL (BigTable). Windows Azure, however, does not offer this integration support for its table storage and this results in additional complexity for the developer (cf. Table 5). Developers have to manually map the (self-defined) implementation of domain entities to a model that can be stored in the Azure Table Storage. This is especially complex when the domain model contains many entity relationships.

Tables in Azure can contain different types of entities and each entity should have a unique primary key. This key consists of a partition key and a row key. The former defines in which partition an entity is stored. Different partitions can be stored on different servers. The row key should be unique within one partition. However, to improve searching for specific entities those keys should be built using well-known information (e.g. non-mutable properties of the entity) instead of generated unique IDs. The only advantage of this complex interface is that it provides developers more control to optimize data storage for specific queries.

Another approach to improve searching is the denormalization of data: data can be stored in multiple tables to facilitate specific search queries. However this complicates the updating of the data enormously: when updating data in multiple tables, consistency should be preserved.

For the implementation of our application case on top of Windows Azure using the table storage, we distinguished two options: (i) store all entities of the same type in one partition, or (ii) use one partition per hotel (together with its address, rooms and bookings). The first option is the easiest to implement: the entity type is used as partition key, and in the row keys of the rooms and bookings we integrate the hotel name and room number to represent the entity relationships. The second option offers a better performance to query and update the rooms and bookings of a particular hotel. These entities are all stored in one partition, and thus require only one transaction. The choice between these or other options depends on which queries are most frequently used. In case of a small set of data, all entities can be stored in a single partition.

We decided to apply the first option for our implementation. Because each hotel has an address, the Hotel and Address entities are stored in one row of the table for hotels. This HotelRow (Listing 6) inherits from TableServiceEntity to indicate it is an entity in the Azure Table Storage. For the other domain entities we made similar Row classes. Based on [49], we developed a custom persistence layer for the mapping from our domain entities (e.g. the Address in Listing 5) and entity relationships to these lower-level entities in the Azure Table Storage. 


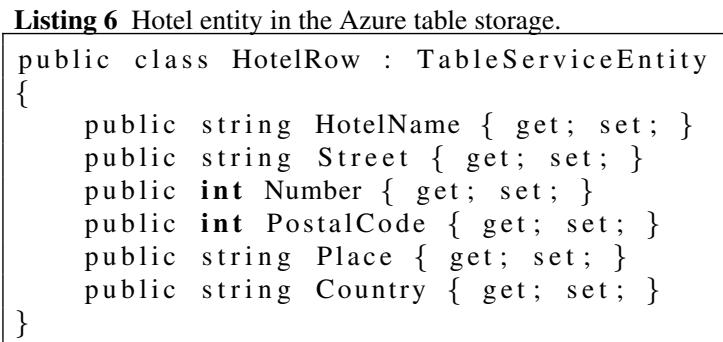

External services and applications. Access to and from external services and applications is possible via SOAP- and REST-based web services. For example, using the WCF framework the developers are able to expose the SaaS application to external applications. Furthermore, the Azure cloud storage provides a RESTful API to enable direct access to data from external systems. As with Force.com, this enhances reusability of applications, data, and services outside Windows Azure.

In addition, the Service Bus is an AppFabric service that provides secure messaging and connectivity capabilities, enabling integration between different applications in the cloud as well as locally. It supports various communication and messaging protocols as well as patterns, ensuring reliable and scalable message delivery. For instance, the Service Bus can be used to create an hybrid application, composing Azure applications with internal components. However, this service is only available at an additional cost. Other announced AppFabric services that support the integration requirement are Integration and Composite App [47,53].

\subsection{Summary}

Compared to Google App Engine and Force.com, Windows Azure offers the best portability support. It is fully compatible with the .NET programming model and frameworks, and also offers extensive integration support. Only the support for the Azure Table Storage is limited. For instance in the case of our hotel booking application, the use of SQL Azure is certainly recommended, because the domain model contains too many relationships.

Force.com is incompatible with the mainstream programming models used in the on-premise implementations of the application case. However, it copes with this issue by providing a wide set of integration capabilities with other programming languages, applications and (cloud) platforms. This enables the decomposition of applications: an application should not be entirely developed on top of Force.com, but it can be integrated with components on other platforms, locally as well as in the cloud.

While Force.com clearly focuses on business applications with its good builtin integration support, Google App Engine aims to facilitate development with good compatibility and to hide complexity. This compatibility, however, has its limitations. Integration support is especially focused on Google's own services, and does not 
provide built-in support to create SOAP- or REST-based web services (in contrast to Force.com and Azure).

\section{Multi-tenancy}

Multi-tenancy is an important enabler for SaaS applications to achieve better operational efficiency $[19,36]$. This section has the following structure for the discussion of each platform. First we describe the general approach applied by the platform to provide multi-tenancy support. Next we discuss for each platform how it addresses the three identified subrequirements, i.e. data isolation, tenant-specific configuration and customization, and tenant-specific application management. Finally we summarize the main conclusions regarding multi-tenancy support by the three investigated PaaS platforms. Table 6 gives an overview of the multi-tenancy support provided by the different investigated PaaS platforms.

Table 6 Overview of multi-tenancy support in the three PaaS platforms.

\begin{tabular}{|c|c|c|c|}
\hline & Data isolation & $\begin{array}{l}\text { Tenant-specific } \\
\text { configuration \& customiza- } \\
\text { tion }\end{array}$ & $\begin{array}{l}\text { Tenant-specific } \\
\text { application } \\
\text { management }\end{array}$ \\
\hline Force.com & $\begin{array}{l}\text { Every tenant has its own } \\
\text { Force.com environment (org) }\end{array}$ & $\begin{array}{l}\text { - App Distribution } \\
\text { - Extension packages } \\
\text { - Custom objects and code }\end{array}$ & $\begin{array}{l}\text { License Manage- } \\
\text { ment Application } \\
\text { (LMA) }\end{array}$ \\
\hline GAE & $\begin{array}{l}\text { - Namespaces API } \\
\text { - Manual mapping of requests } \\
\text { to namespace }\end{array}$ & $\begin{array}{l}\text { - Dependency Injection } \\
\text { (Guice Provider) } \\
\text { - Manual configuration man- } \\
\text { agement }\end{array}$ & $\mathrm{n} / \mathrm{a}$ \\
\hline Azure & $\begin{array}{l}\text { No support: } \\
\text { - Manual data isolation } \\
\text { - No built-in tenant context } \\
\text { - Manual mapping of requests } \\
\text { to tenant context }\end{array}$ & $\begin{array}{l}\text { - Only if data isolation is en- } \\
\text { sured } \\
\text { - Dependency Injection } \\
\text { (Ninject Provider) } \\
\text { - Manual configuration man- } \\
\text { agement }\end{array}$ & $\mathrm{n} / \mathrm{a}$ \\
\hline
\end{tabular}

\subsection{Force.com}

Force.com offers built-in support for data isolation as well as tenant-specific configuration and customization by providing each tenant with a separate environment. In addition, it supports the tracking of tenant usage via the License Management Application (LMA).

\subsubsection{General approach.}

Development and deployment (production) on Force.com typically takes place in an environment or organization, frequently shortened to org [38]. Such an environ- 
ment provides a number of features for applications within that environment and ensures all tenant-specific data (including metadata) are separated from other orgs. The Force.com platform offers two ways to deliver applications depending on who the SaaS consumers are:

- Single Org model: the application runs in the org of the SaaS provider. This model is especially applied by organizations to build applications for internal use, only accessible by the own employees (i.e. SaaS provider and SaaS consumer are the same organization).

- Distributed Org model: the application is packaged and installed by SaaS consumers in their own Force.com environment (or org). This model is suitable for developing, running and distributing a multi-tenant SaaS application.

Since we want to offer the hotel booking application as a multi-tenant SaaS application, only the distributed org model is relevant for this paper. All components that make up the application (i.e. application metadata) are bundled together in a package. This package can be made available to the Force.com market via AppExchange (part of the App Distribution core resource). This is a central directory service that enables tenants to find and install applications.

Packages exist in two forms. The unmanaged package form is intended for the distribution of applications or components, together with the source code. By installing an unmanaged package the tenant receives its own copy, which can be modified independently from the creator of the package. Managed packages, however, obfuscate most of the source code and permit package creators (i.e. SaaS providers) to offer upgrades.

\subsubsection{Data isolation.}

By providing each tenant with a separate environment or org, Force.com inherently supports data isolation. When a tenant installs a reference to an application in its org (e.g. the hotel booking application), the application metadata is copied into its org. This application metadata is separately stored in the multi-tenant database, together with the tenant-specific metadata (configuration and customizations) and the application data (see Fig. 3).

Each org also specifies the different users and user groups of the tenant, as well as their roles and permissions. Login names of users are automatically mapped to a specific tenant and org. The creator and owner of the org receives the tenant administrator role, responsible for the management of the installed applications (e.g. configuration) and user management.

\subsubsection{Tenant-specific configuration \& customization.}

The metadata-driven architecture of the Force.com platform enables tenants to configure and customize the application to fit their specific requirements. To offer a customizable SaaS application as described in the case study, the SaaS provider can provide extension packages. These enable the creation of new functionality on top of a base managed package. Extension packages can also be managed or unmanaged. 
By means of these extension packages SaaS providers are able to offer multiple variants of their application. In the implementation of our hotel booking application, the price calculation strategy based on the customer profile can be implemented as an extension package. The tenant administrator can configure the application by selecting and installing the necessary extension packages.

In addition, the tenant administrator can further tailor the application by adding custom data objects and Apex code (i.e. tenant-specific metadata), or by integrating the application with other applications installed into his org. As mentioned above, managed packages obfuscate most of the source code. Therefore tenant-specific customizations are limited to a class of changes that do not hinder seamless upgrades (by the SaaS provider) from occuring.

The Force.com platform almost transparently supports the development and deployment of multi-tenant applications via the App Distribution. However, the SaaS provider has to take several trade-offs into account. Depending on which features of the platform are used in the application, tenants should have a specific Force.com edition. This can be partially solved by offering different versions via extension packages. In addition, some features cannot be packaged (yet) and require manual configuration by the tenant administrator.

\subsubsection{Tenant-specific application management.}

The License Management Application (LMA) of Force.com facilitates tracking of installation and upgrades of the managed packages created by the SaaS provider. The LMA enables the SaaS provider to apply licensing on managed packages to control how many end users in a tenant organization can access the package and for how long. LMA is available as an application in AppExchange. Force.com, however, does not support tenant-specific performance and resource consumption monitoring.

\subsection{Google App Engine}

Built-in multi-tenancy support provided by Google App Engine (GAE) is limited to data isolation. Tenant-specific configuration and customization can be achieved by using dependency injection (as explained below), but this requires additional implementation work by the SaaS provider. GAE also lacks support for tenant-specific application management.

\subsubsection{General approach.}

To support the development of multi-tenant applications, GAE provides the Namespaces API. A namespace is set globally for each tenant using the namespace manager. More specifically, the namespace corresponds to a unique tenant ID and represents the context containing the information of the tenant linked to the current request. The GAE APIs that support namespaces will automatically use this current namespace (e.g. datastore and memcache). However, it is still possible to change the current namespace locally while processing a request. Wrong usage can lead to unintended data leaks between different tenants. 


\subsubsection{Data isolation.}

The Namespaces API automatically supports the partitioning of data across tenants by specifying a unique namespace for each tenant. Thus, by assigning a unique namespace to each tenant (e.g. the tenant ID), data isolation is preserved. Internally, the datastore and memcache store, update and query data based on the current namespace. Global data, accessible by all tenants, can be stored in a separate namespace. However, the mapping of an incoming request to a specific namespace does not occur automatically. A practical solution is presented in the following paragraph.

In addition, GAE lacks support for proper user management of a multi-tenant application, for example to assign the tenant administrator role. The Google Accounts and the Users service are not sufficient to distinguish the tenant administrator from normal end users. Separate roles only exist for the SaaS provider (administrator and developer). Therefore SaaS developers should develop their own user management system (as part of the application) to allow the tenant administrator to configure the application. This is certainly necessary when access control is an important requirement.

Mapping to namespace. For each request we have to retrieve the tenant ID and set the namespace for that request, ensuring that the request will be processed in the context of that tenant (tenant-specific data and configuration). For the implementation of the hotel booking application on top of GAE, we determine the tenant ID based on the used Google Apps domain (URL) to access the SaaS application. Applications can be deployed to any Internet domain owned by the SaaS provider and registered in his Google Apps account. Each domain then corresponds to a namespace.

Incoming requests with a specific domain name (i.e. tenant ID) should be mapped to a namespace. Therefore we use Java Servlet Filters (cf. [32]). A filter dynamically intercepts requests and responses, enabling the inspection or transformation of the contained information. For every request our TenantFilter (Listing 7) checks whether the namespace is already set. If not, the tenant ID is retrieved from the Google Apps domain (line 17). Next, the namespace is set to this tenant ID (line 19).

\subsubsection{Tenant-specific configuration \& customization.}

Google App Engine does not support tenant-specific configuration and customization of applications. In our implementation, we achieved it by using Google's dependency injection framework, Guice (v3.0) [33]. We discuss this in depth in the following paragraph. The tenant-specific configuration settings are stored as part of the tenant data. However, in contrast to Force.com, there is no support for tenant administrators to manage this configuration themselves (via a configuration interface). Similarly, GAE lacks support for the SaaS provider to develop and manage the different features of an application. Both require additional development by the SaaS provider.

Tenant-aware dependency injection. Dependency injection (DI) is a design pattern where component dependencies are managed by an injector, rather than relying on the 


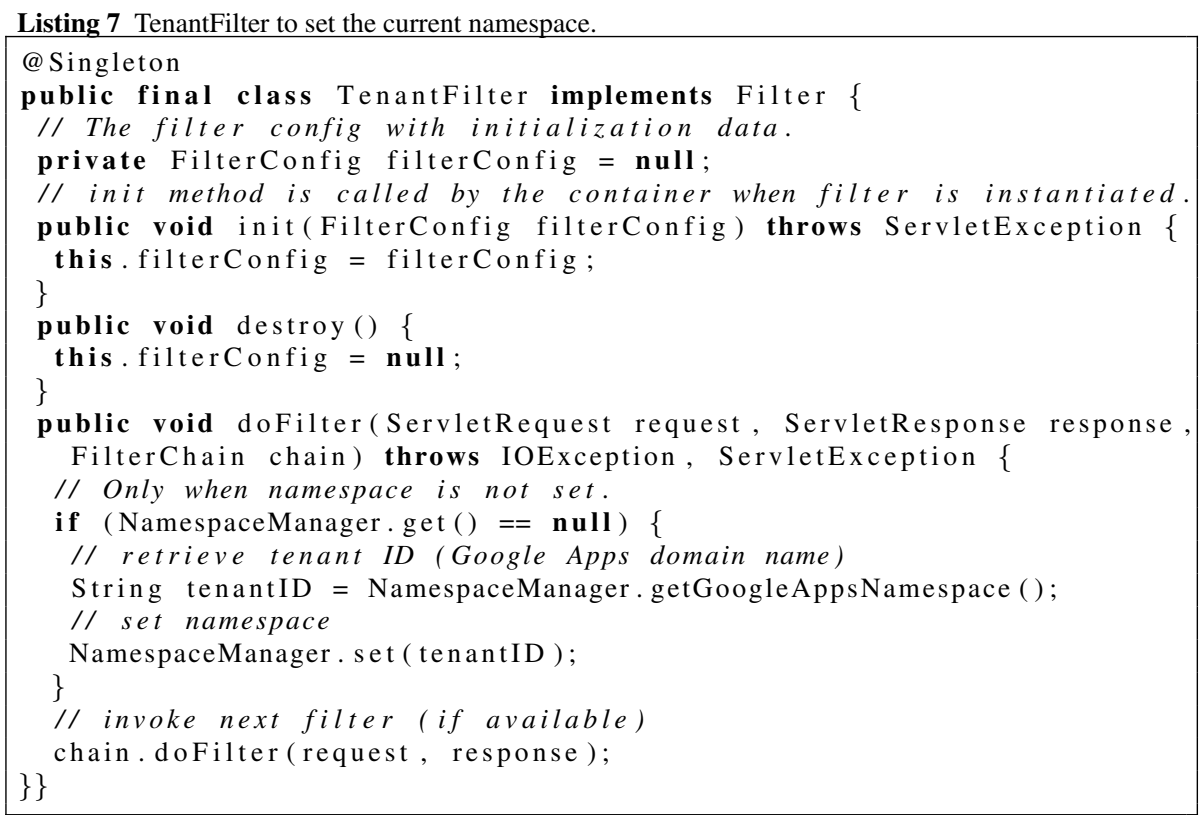

component itself to fill in the dependency [28]. By using such a DI framework [28] on top of GAE, we are able to support tenant-specific customization. Instead of instantiating the feature implementations directly in the application, the flow of control is inverted: the life cycle management of feature implementations is controlled by a dependency injector. This injector binds dependencies in the application to a specific implementation component. In the case study, this means that the dependency of the hotel booking application on IPriceCalculatorStrategy is bound to one of the available feature implementations (cf. DefaultPriceCalculator and CustomerProfileBasedPriceCalculator in Fig. 2).

Originally the configuration of a GAE application is stored in the web.xml file, which is shared by all tenants. By means of Guice, the configuration can be dynamically loaded. However, the dependency injector is shared by all tenants. This hinders the isolated execution of tenant-specific customizations: all dependencies are set globally and any modification would affect all tenants. This is a general problem with dependency injection because it does not support activation scopes.

To solve this issue, we added an extra level of indirection. Instead of injecting features, we inject a Provider for that feature. This way the servlets have a dependency to a provider of a feature instead of to the feature itself. However, the customizations that can be performed this way are limited to switching between implementations of an interface or abstract class. Listing 8 presents the PriceCalculatorProvider that decides based on the tenant-specific configuration which feature implementation of the IPriceCalculatorStrategy feature should be selected: normal price calculation or with discounts based on the customer profile (lines 19-21). This fea- 
ture implementation is then instantiated (line 29). If no tenant-specific configuration for this feature is set, the default option is retrieved and instantiated (lines 24-27). The instantiated tenant-specific feature implementation is stored in the (namespaceenabled) Memcache to improve performance.

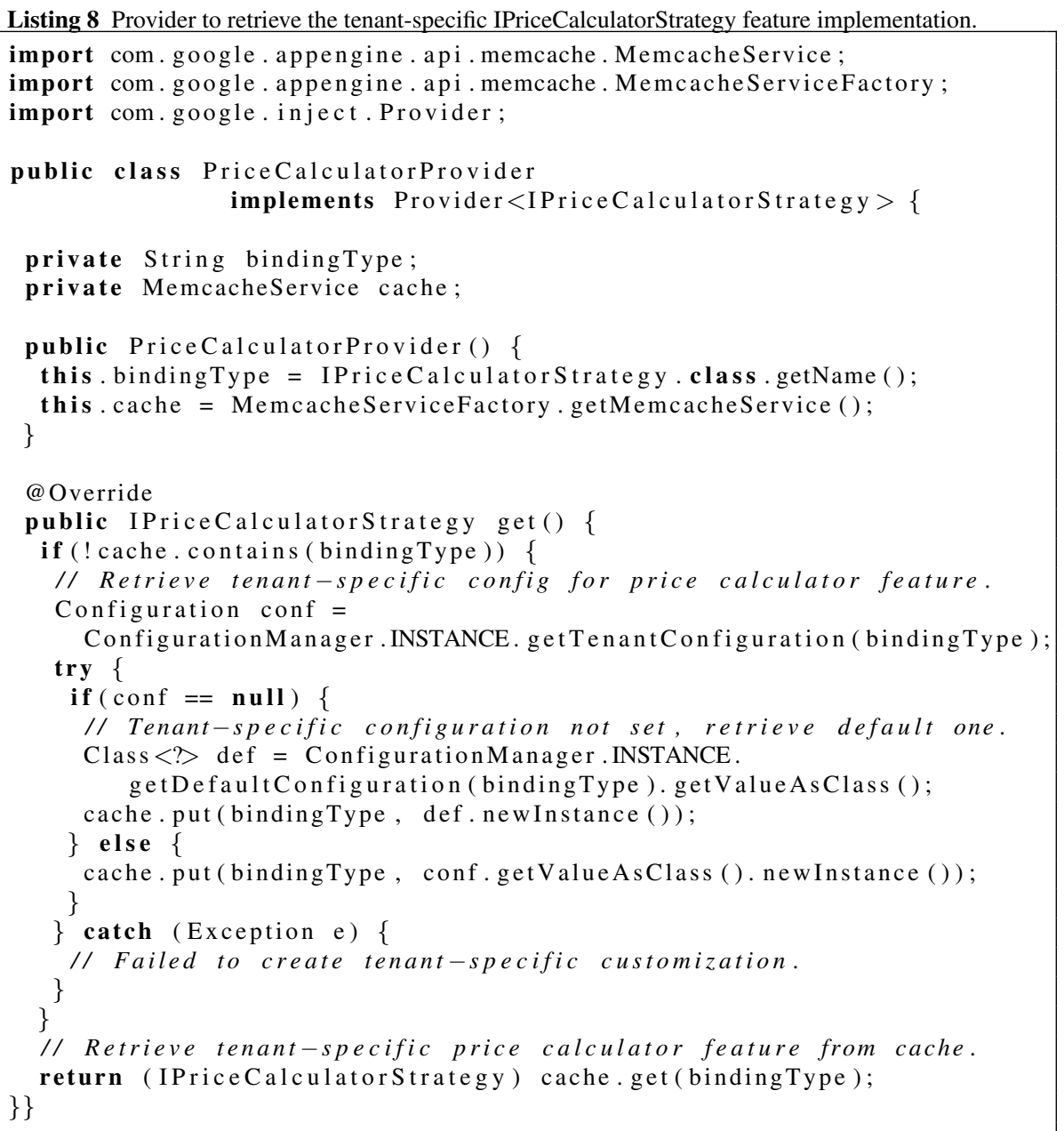

\subsubsection{Tenant-specific application management.}

GAE applications can only be monitored on a per application basis, which is too coarse-grained for multi-tenant SaaS applications. The Java SDK of GAE includes a suite of tools for measuring the performance of applications, called Appstats. Appstats integrates with the application using a servlet filter to record events, and provides 
a web-based administrative interface for browsing statistics. To enable tenant-specific monitoring, the SaaS provider will have to manually filter and record the incoming requests per tenant, as far as this is possible. Additional implementation is also required to support billing and to track which features are used by the different tenants.

\subsection{Windows Azure}

Windows Azure offers no support for multi-tenancy. SaaS providers will have to provide this themselves. If data isolation can be ensured, tenant-specific configuration and customization can be achieved, for example by using dependency injection (cf. Google App Engine).

\subsubsection{General approach.}

Windows Azure does not provide support for developing multi-tenant applications. This lack of built-in support complicates the development and forces the SaaS provider to implement its own multi-tenancy strategy. In [19] and [49] (Part 2, Chapter "Hosting a Multi-tenant Application on Windows Azure") different options for implementing a multi-tenant application are presented, but this is limited to data isolation.

\subsubsection{Data isolation.}

As part of the requirements of data isolation, we identified that two main components are required: (i) a tenant context containing the information of the tenant linked to the current request (via a unique tenant ID) (cf. namespace in GAE), and (ii) multitenant data storage. Neither is provided by Windows Azure. The same applies to tenant-aware user management (e.g. no tenant administrator role). The AppFabric Access Control service also does not have built-in support for multi-tenant applications. Like in GAE, this should be implemented by the SaaS provider. In the following paragraphs we present our solution to obtain a tenant context and a multi-tenant data storage.

Tenant context. Similar to Google App Engine, a filter is required to intercept the incoming requests and to set the current tenant context. It is also possible in Azure to assign a separate domain to each tenant of the SaaS application. However, custom domains are not supported when using SSL, because the IIS web server can only have one SSL certificate associated with a port (default port 443). In this case the tenant ID in Windows Azure is determined based on the context path of the URL: https://<azure-account>. cloudapp. net/<application $>/<$ tenant-id>/*.

For the hotel booking application, we acquire the tenant ID from the context path via the MvcApplication. RegisterRoutes method provided by the MVC framework and add it to the request context (see lines 6-10 in Listing 9). Each route consists of a route name, a template URL, and a list of default parameters. The presented route sends the requests to the BookingController, calling the Index() method. In the 


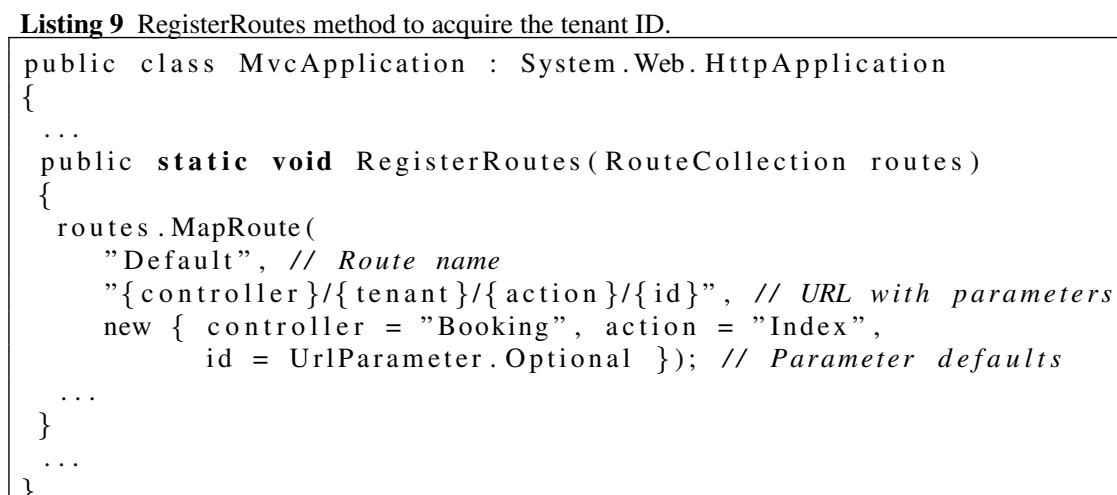

same way as the controller and action parameters are added to the request context, the tenant ID is passed through with the "tenant" key.

The next step consists of setting the current tenant context. To represent the tenant context we use TenantController, an abstract MVC controller class with the filter method OnActionExecuting. All other controllers (e.g. the BookingController) inherit from this TenantController and therefore have access to the tenant context. Listing 10 shows the TenantController: it retrieves the tenant ID from the request context by means of the "tenant" key (lines 20-22), verifies the tenant ID (lines 26-27), and then sets the current tenant (line 25). The TenantStore refers to the table storage containing information about all tenants. This approach is based on the Windows Azure Architecture Guide (Part 2) [49], but it is limited to MVC applications. Alternatively, a similar approach with namespaces like GAE can be used: an application-wide tenant context manager storing the tenant context of the current request.

Multi-tenant data storage. A multi-tenant data storage can be obtained by applying filters that intercept the calls to the storage API and inject the tenant ID from the associated tenant context. In addition, comparable interceptors are necessary for the caching service (distributed in-memory storage). This forms a tenant-aware layer on top of the data storage, which makes abstraction of the chosen data isolation strategy.

In [20], three distinct approaches for creating multi-tenant data architectures are identified. A common solution on Azure to achieve tenant-aware data isolation is to assign a separate SQL Azure database for each tenant. This is a simple approach for the SaaS provider, but rather expensive for the tenant. The selection of the right data isolation strategy also depends on the kind of data (e.g. number of relationships between the data entities), and the kind of queries on these data. We integrated tenantaware data isolation into our implementation of a custom persistence layer for Azure Table Storage. Isolation is achieved by adding the tenant ID (retrieved from the tenant context) to the partition key of each entity. 


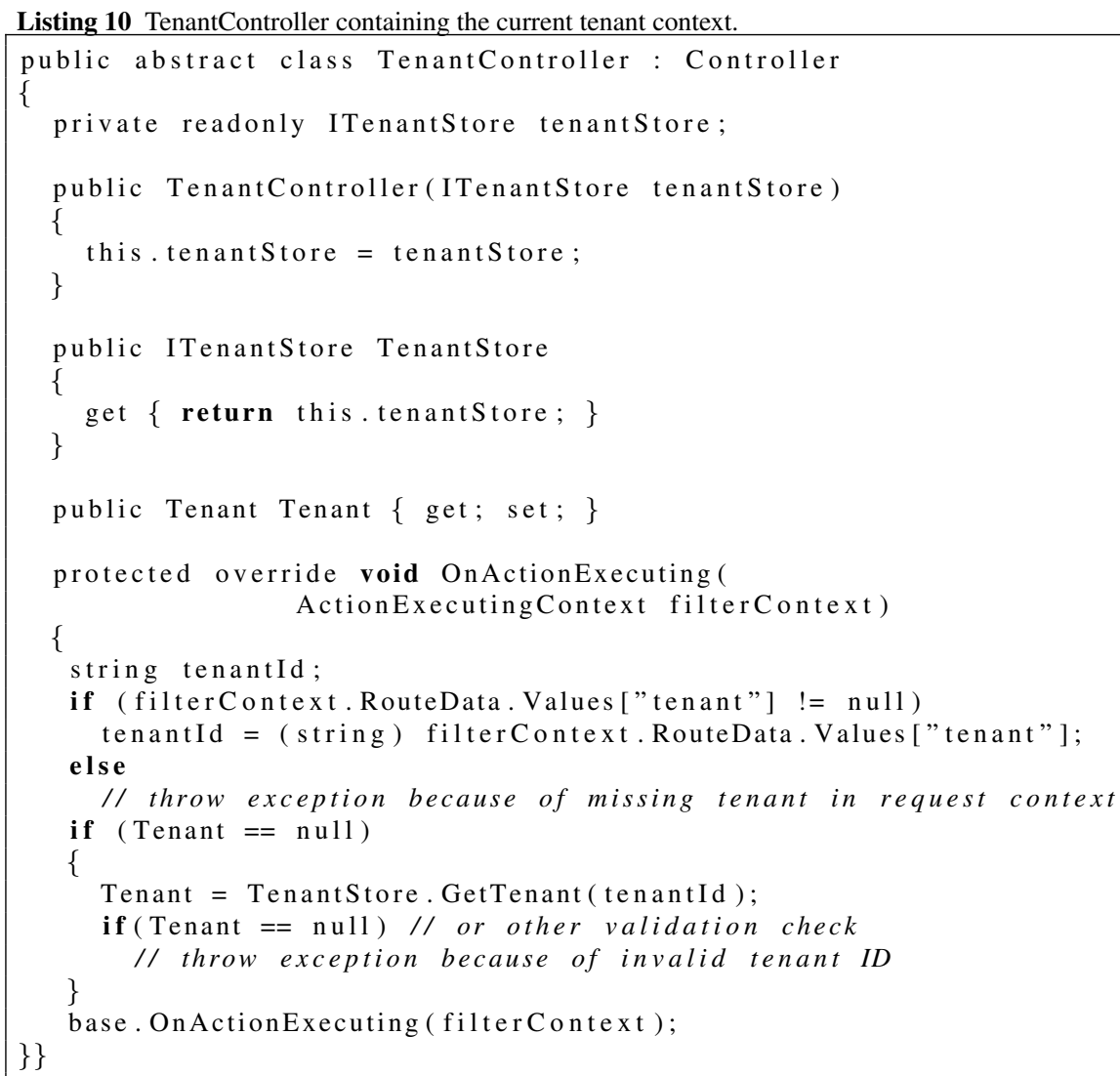

\subsubsection{Tenant-specific configuration \& customization.}

Windows Azure offers no support for tenant-specific configuration and customization. However, if data isolation is ensured and the tenant IDs can be retrieved, for instance using the approach described above, it is possible to achieve tenant-specific customization via dependency injection (cf. our approach on GAE). Azure also lacks support for tenant administrators to manage the tenant-specific configuration and for SaaS providers to develop and manage the different features. The SaaS provider should develop and provide this, preferably as a reusable framework. The next paragraph describes our approach for tenant-specific customization using the Ninject dependency injection framework (v2.2) [42].

Tenant-aware dependency injection. To achieve tenant-specific customization using dependency injection, we have applied the same approach as on Google App Engine. The Ninject dependency injection framework is, like Guice, not tenant-aware. However, Ninject uses by default a provider, the StandardProvider. Each depen- 
dency is handled by this provider, and therefore there is no direct binding between a type (i.e. interface) and the injected implementation. A provider can decide based on a IContext parameter which implementation should be injected. This offers the perspective to make Ninject tenant-aware by passing the tenant ID via this context parameter. It requires additional development by the SaaS provider to ensure (i) the right tenant ID is added to the context parameter, perhaps by means of a dedicated IContext implementation, and (ii) the tenant ID is used by a tenant-aware provider to return the right customization based on the tenant-specific configuration.

Listing 11 presents our solution for tenant-specific customizations on top of Azure. We provided a PriceCalculatorModule, which is used to assign PriceCalculatorProvider as the custom, tenant-aware provider for injecting the price calculation feature on a per tenant basis (lines 13-14). This PriceCalculatorProvider first tries to retrieve the tenant-specific configuration for the price calculation feature, or when not available, the default one (lines 26-32). Next, it can retrieve and instantiate the appropriate implementation for the IPriceCalculatorStrategy feature (lines 34-35). Another approach for tenant-specific customizations using MVC and Ninject is presented in [27], but it is not applied on top of a cloud platform.

\subsubsection{Tenant-specific application management.}

Similar to Google App Engine, Windows Azure only supports application-specific monitoring, which is too coarse-grained. To fullfil the requirement of tenant-specific application management, SaaS providers will have to implement this facility themselves.

\subsection{Summary}

Force.com is the only PaaS platform that provides built-in support for data isolation as well as tenant-specific configuration and customization. Concretely, the SaaS developer does not have to write additional code to support multi-tenancy (Table 7). The only condition is that both the SaaS provider and the tenants have an account (org) on Force.com. Multi-tenancy support on Google App Engine is limited to data isolation, while Windows Azure lacks any support for the development of multi-tenant SaaS applications. If data isolation is ensured (via Namespaces API or manually), we demonstrated that dependency injection can be used to support tenant-specific customizations, but in a limited way compared to Force.com (for instance, tenants cannot add custom code). In addition, the SaaS developers should implement support for tenant-aware user management (e.g. to assign the tenant administrator role), and for managing the different features and configurations (i.e. configuration interface). This development effort is considerable, at least 600 to 750 lines of code to achieve a minimal support for multi-tenancy (Table 7). In the case of GAE, the Namespaces API enables a rather modularized approach to add multi-tenancy support. On Azure, however, the additional code for multi-tenancy support is scattered across the different tiers of the application, adding up to the development and maintenance complex- 
Listing 11 Structure of the PriceCalculatorModule and the PriceCalculatorProvider on Azure.

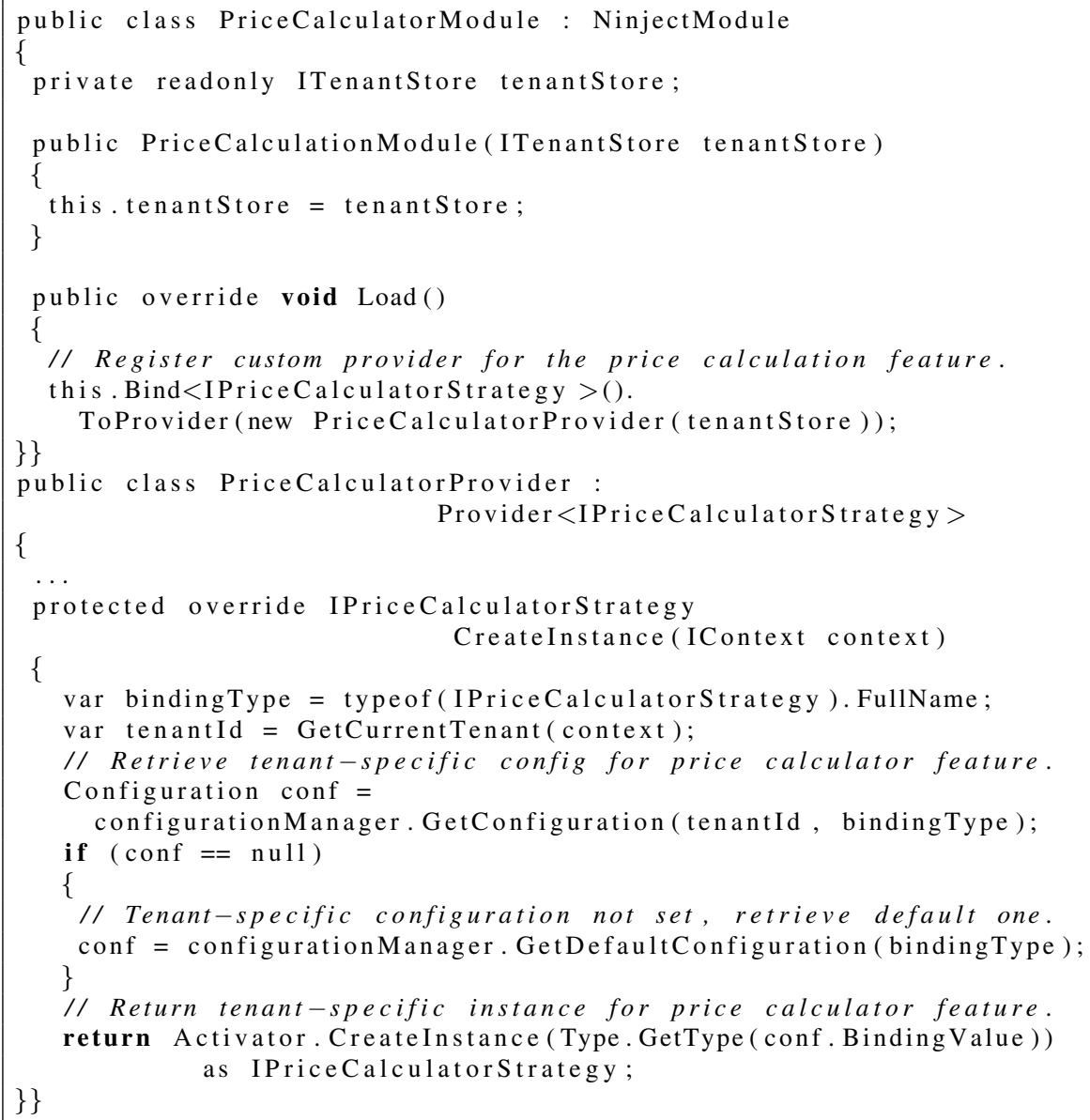

ity. Finally, none of the investigated PaaS platforms fully addresses the tenant-specific application management requirement.

\section{Tool Support}

Tool support plays an important role to achieve smooth adoption of PaaS platforms. We discuss for each platform how it tackles the three identified subrequirements concerning tool support: (i) a development environment, (ii) a local development server and storage, and (iii) a testing framework. Table 8 presents an overview of the tool support provided by the different investigated PaaS platforms. 
Table 7 Overview of the code size (in lines of code) of the full implementations of the hotel booking application on top of the different PaaS platforms. The last column indicates the ratio of the implementation for multi-tenancy support compared to the full implementation. Note: the code size of the Force.com implementation also includes the metadata that is generated by using the point-and-click interface.

\begin{tabular}{|c|c|c|c|c|}
\hline & Type & \# files & \# lines of code & $\%$ multi-tenancy \\
\hline Force.com & $\begin{array}{c}\text { Apex } \\
\text { XML (generated metadata) } \\
\text { VisualForce }\end{array}$ & $\begin{array}{c}8 \\
24 \\
6\end{array}$ & $\begin{array}{l}753 \\
615 \\
229\end{array}$ & / \\
\hline GAE & $\begin{array}{c}\text { Java } \\
\text { JSP } \\
\text { XML (config) } \\
\text { HTML }\end{array}$ & $\begin{array}{c}41 \\
16 \\
3 \\
4\end{array}$ & $\begin{array}{c}1830 \\
616 \\
72 \\
24\end{array}$ & $\pm 29 \%$ \\
\hline Azure & $\begin{array}{c}\text { C\# } \\
\text { ASP.NET Web Page } \\
\text { XML (ASP.NET config) }\end{array}$ & $\begin{array}{cc}\text { SQL } & \text { Table } \\
56 & 69 \\
23 & 23 \\
9 & 9\end{array}$ & $\begin{array}{cc}\text { SQL } & \text { Table } \\
1791 & 2509 \\
919 & 919 \\
197 & 187\end{array}$ & $\pm 21 \%$ \\
\hline
\end{tabular}

Table 8 Overview of tool support for the three investigated PaaS platforms.

\begin{tabular}{|c|c|c|c|}
\hline & $\begin{array}{l}\text { Development environ- } \\
\text { ment }\end{array}$ & $\begin{array}{l}\text { Local development } \\
\text { server \& storage }\end{array}$ & Testing framework \\
\hline Force.com & $\begin{aligned} \text { - Web interface (main) } \\
\text { - Force.com IDE } \\
\text { (Eclipse plugin) }\end{aligned}$ & Always remote & $\begin{array}{l}\text { - Apex Unit tests } \\
\text { - } 75 \% \text { of all Apex code } \\
\text { needs to be covered }\end{array}$ \\
\hline GAE & $\begin{aligned} \text { - Eclipse plugin } \\
\text { - App Engine SDK + } \\
\text { Apache Ant }\end{aligned}$ & $\begin{array}{l}\text { Development web server } \\
\& \text { datastore simulation }\end{array}$ & $\begin{array}{l}\text { - JUnit \& others } \\
\text { - Google App Engine test- } \\
\text { ing utilities }\end{array}$ \\
\hline Azure & $\begin{array}{l}\text { - Visual Studio plugin } \\
\text { - Windows Azure SDK }\end{array}$ & $\begin{array}{l}\text { - Compute \& storage } \\
\text { emulator } \\
\text { - SQL (Express) Server }\end{array}$ & Visual Studio test tools \\
\hline
\end{tabular}

\subsection{Force.com}

The main development environment of Force.com is a web interface and it does not provide a local development server or storage. Unit tests are written in the Apex language. To develop and test applications on top of Force.com, a (free) Development Environment (DE) account is required.

\subsubsection{Development environment.}

Force.com offers two development environments: an online development environment inside the browser and an IDE based on Eclipse. The web interface supports SaaS developers with creating custom objects and workflows, modifying business objects, and writing Apex code. In addition, tenant administrators are able to administer the application (e.g. configuration, user management and security controls). Compared to the web interface the Force.com IDE is not mature yet: the provided functionality is limited to writing Apex code and Visualforce pages (UI), and some features do not work well, for instance auto-completion of SOQL statements or Vi- 
sualforce code as well as refactoring. Therefore, SaaS developers are often forced to use the web interface instead of the IDE they are familiarized with.

\subsubsection{Local development server \& storage.}

To make the application resources accessible via the web interface, all code and metadata is synchronized with the Development Environment (DE) account on the server. The platform does not provide a local development server or storage: validation and execution only occur at the server side. This has as consequence that the IDE is less responsive than the web interface (each 'save' operation results in a synchronization) and prevents integration with the Eclipse debugger. In addition, this constant synchronization with the server complicates collaboration between multiple developers because there is no versioning support. This issue can be solved by using a Partner Development Environment which is a master repository environment to manage all the source code; each developer can check out to his own DE.

\subsubsection{Testing framework.}

Force.com requires the creation and execution of unit tests to deploy Apex code to a production environment. These test classes and methods are written in the Apex language and verify whether a particular piece of code is working properly. At least $75 \%$ of the Apex code should be covered before deployment is possible. This encourages test-driven development and improves correctness of the Apex code. An example of a test method for the CheckBookingPeriod trigger is presented in Listing 12. Notice that the approach is similar to unit tests in common programming languages.

\subsection{Google App Engine}

Google App Engine comes with an Eclipse plugin, a local development web server and datastore simulator, and testing utilities to support the writing of unit tests. A (free) Google account is only required to deploy the application in the cloud.

\subsubsection{Development environment.}

To ease the development of Java App Engine applications, Google offers a plugin for Eclipse (Java EE version) to create, test and upload applications from within Eclipse. In contrast to the Force.com IDE, this plugin works smoothly. There is only a small issue with the (rather frequent) updates of the GAE libraries: when collaborating with multiple developers this can lead to inconsistencies.

\subsubsection{Local development server \& storage.}

The Google App Engine SDK includes a development web server that simulates the GAE Java runtime environment and the supporting services such as the datastore and the users service. While the Force.com IDE sends all code to an external server, this 


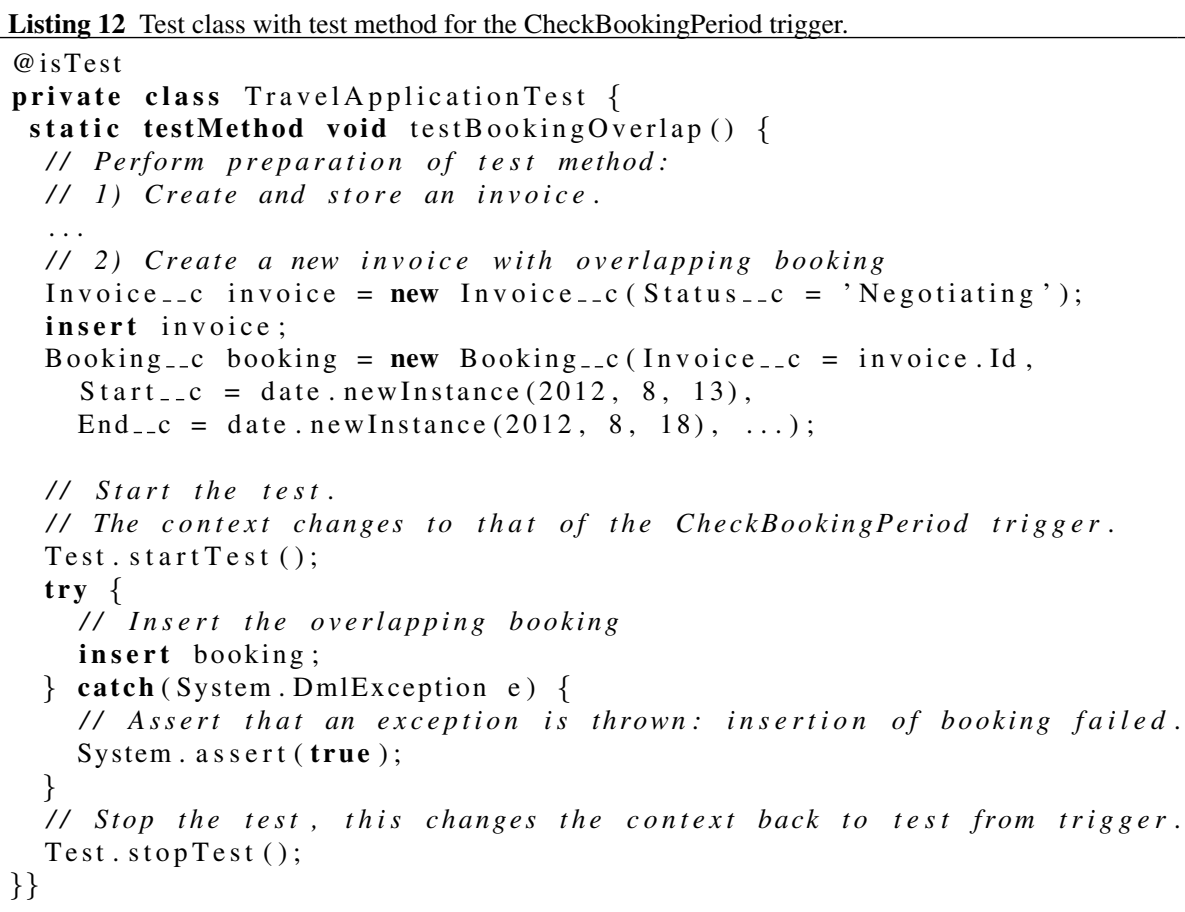

development server allows to extensively test GAE applications locally. The server can be executed in the Eclipse debugger via the Google plugin, or via the command line (using Apache Ant). However, the behaviour of applications running on the development server does not always correspond to their behaviour on top of GAE. For example, objects that are stored using the Memcache service should be serializable, however this is not required by the development server. Applications that run on the development server, therefore not necessarily work on top of GAE. Force.com does not have this problem, because it is always validated and executed on the (remote) Force.com platform.

\subsubsection{Testing framework.}

The SDK also contains testing utilities to support the writing of unit tests that have dependencies on Google App Engine services. The LocalServiceTestHelper class is a helper class that handles the setup of the environment. To configure the local GAE services, it requires a LocalServiceTestConfig instance as argument for each used service. For example, when using the datastore service, a LocalDatastoreServiceTestConfig instance should be passed as argument to the constructor. The GAE testing utilities are not tied to any specific testing framework, so it is possible to use JUnit, a widely used unit testing framework for Java. In contrast to Force.com, 
unit tests are not mandatory. Listing 13 shows the outline of a JUnit test case with dependencies on the Datastore and Memcache services.

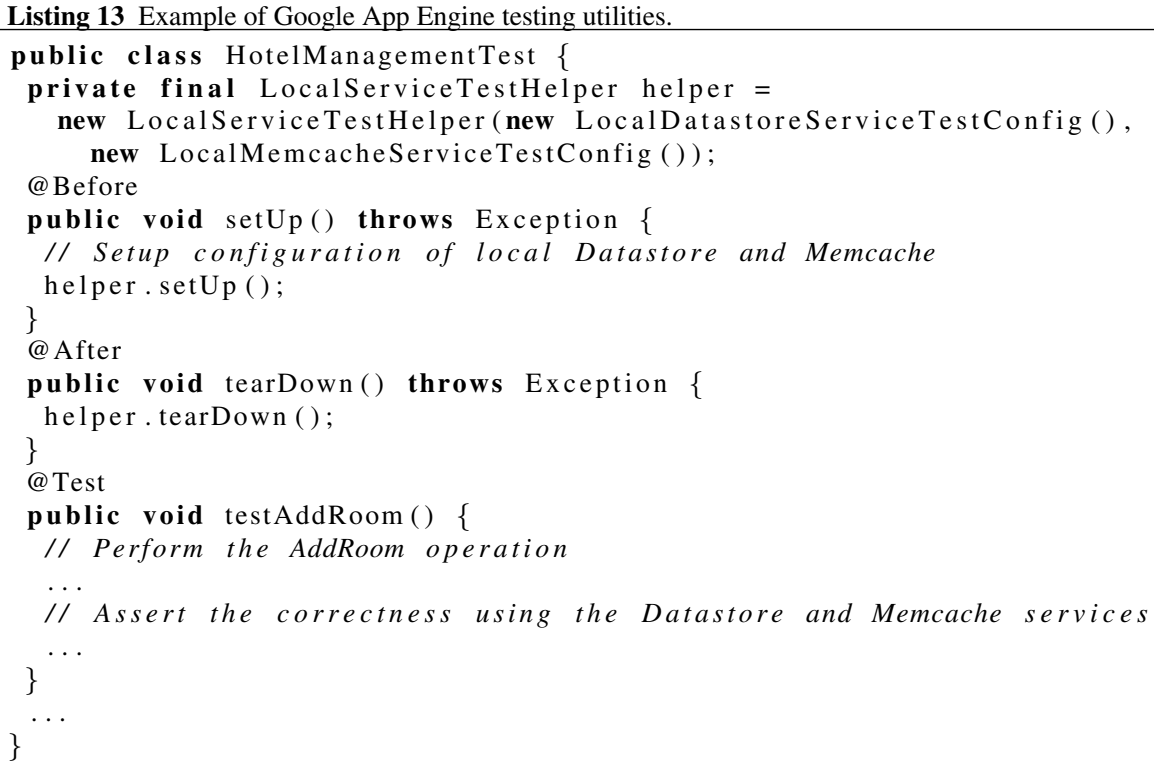

\subsection{Windows Azure}

Development for Windows Azure is supported by a Visual Studio plugin and a compute and storage emulator. Visual Studio provides by default the necessary testing tools to write unit tests. Deployment of applications on Windows Azure requires an Azure account.

\subsubsection{Development environment.}

The Windows Azure SDK is available in a standalone version or can be installed together with the Windows Azure Tools, a plugin for the Visual Studio IDE. These tools are also available for Eclipse to support Java development for Windows Azure. Moreover, it is possible to import existing .NET projects into a new Azure project. This supports the migration from an on-premise implementation to Azure.

\subsubsection{Local development server \& storage.}

The Windows Azure SDK offers a compute and storage emulator. This enables SaaS developers to test their Windows Azure applications locally without an Azure account. There exists no local SQL Azure database, but applications that access SQL 
(Express) Server locally will largely work unchanged with SQL Azure. For example, distributed transactions across multiple databases are not supported by SQL Azure. Other differences are less important and only apply in exceptional cases. In contrast to Force.com and Google, Microsoft does not provide a free developer account to deploy and test applications in the cloud.

\subsubsection{Testing framework.}

To create unit tests the existing testing tools provided by Visual Studio can be reused. However, there is no direct support, like GAE provides, to test an application against the storage tools of the Azure SDK. To effectively write unit tests for classes that interact with Windows Azure Storage, it should be possible to mockup the calls to the storage service. This can be achieved by writing a wrapper interface for WindowsAzure. StorageClient that can be reused by all classes and applications interacting with the storage service. This has been implemented in the sample application for part 2 of the Windows Azure Architecture Guide [49]. Another option is to setup the emulator with a default test configuration, like the Google App Engine test utilities do.

\subsection{Summary}

All investigated platforms provide good tool support in the form of a plugin for a commonly used IDE. However, in the case of Force.com the IDE is not sufficient to develop an entire application, but only supports Apex and Visualforce development. Google App Engine and Windows Azure also allow developers to use a standalone version of the SDK in combination with the command line. Another difference between Force.com and the two other PaaS platforms is the lack of a local development server. While GAE and Windows Azure support local testing of cloud applications, Force.com requires that all code is synchronized on their servers. Both differences are disadvantages caused by the metadata-driven architecture of the Force.com platform. Finally, all platforms support unit testing that is similar to the on-premise approach.

\section{Discussion \& Challenges}

In this section we discuss the different requirements for developing SaaS applications in the context of the different PaaS categories and relate these requirements to our results. Further, we discuss the impact of the specific case study on our findings and we briefly analyze the impact of our implementation decisions on these results. Finally, we itemize a set of open challenges that we have identified.

\subsection{Application of results to PaaS categories}

The main challenge for SaaS providers is to select the most appropriate PaaS platform (or category of PaaS) for their SaaS application. Our comparison has demonstrated 
that none of the investigated cloud platforms stands out above the others with respect to all three requirements concerning support for SaaS development.

Based on the results of this experience report, we can conclude that tool support is not a key differentiator with respect to SaaS development. Most PaaS platforms already offer good tool support in the form of a plugin for one of the common IDEs, and integration support with unit testing frameworks (e.g. JUnit). A point of attention is that the behaviour of the local development server should correspond with the cloud environment to enable developers to fully test the SaaS application. For example, we noticed this is not always the case with GAE.

Consequently, the selection between PaaS platforms can be reduced to the choice between portability and multi-tenancy support. The three PaaS platforms (of different categories) that we compared all relate differently to the portability and multi-tenancy requirements (see Fig. 6). The metadata-driven PaaS platforms (e.g. Force.com) are clearly the winner with respect to multi-tenancy. However, if portability takes precedence, PaaS category 1 is the best option, with the platforms of category 2 as runnerup. The following paragraphs discuss portability and multi-tenancy support for each of the PaaS categories.

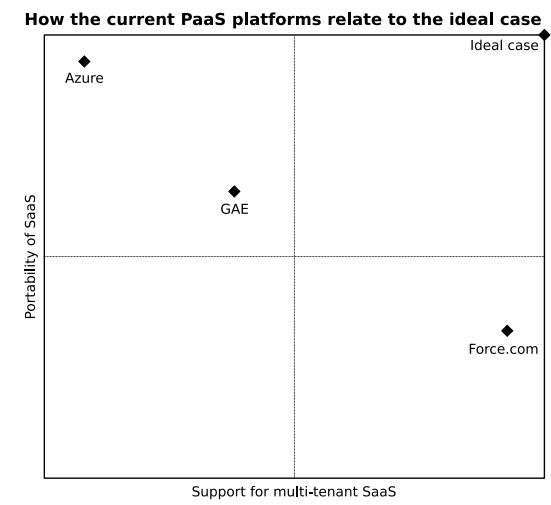

Fig. 6 Overview of how the three representative PaaS platforms that we compared relate to the portability and multi-tenancy requirements. The data points are based on the different scores as defined in Section 2.3. The right top corner represents the ideal case of a PaaS platform that fully addresses both requirements. This figure clearly reveals the gap between the existing platforms and the ideal case.

PaaS category 1. The main goal of PaaS category 1 is clearly to support the provider's customers and developers in the migration from the on-premise to the cloud model. To achieve this goal, these PaaS providers try to support the standard APIs that are used in popular enterprise application servers and middleware frameworks. This is reflected by a high degree of portability, as clearly illustrated by Windows Azure with its high level of code reuse (Table 5 and Fig. 6). Typically, using PaaS platforms of this category results in code reuse of at least 90-95\%. Moreover, the use of standard APIs not only facilitates the migration from on-premise to PaaS, but also between PaaS platforms of this category. Obviously, the latter is only valid as long as the same programming language and similar APIs are supported. 
Because these PaaS platforms are based on existing solutions with standard APIs, they are often also available as a private cloud platform. For example, Red Hat OpenShift [63] (Java EE and JBoss applications) and Cloud Foundry [80] (Spring applications) are two open source PaaS platforms with both public and private cloud offerings. This way, SaaS providers have the option to keep the execution of their SaaS applications in-house.

To improve performance and scalabiliy, these PaaS platforms are extended with typical cloud services, such as scalable storage and distributed caching. However, support to integrate with these services is limited or lacking, and this requires a significant amount of additional development by the SaaS provider. For example, we had to build a custom persistence layer to map our domain model to row entities that could be stored in the Azure Table Storage (Table 5). Some platforms of category 1, for instance Amazon Elastic Beanstalk [3], provide vendor-specific SDKs to make abstraction of the lower-level APIs for scalable storage services and to reduce the development overhead. However, such an approach compromises the portability.

Support for developing multi-tenant applications is completely lacking in PaaS category 1 . The SaaS provider has to build the multi-tenancy support from scratch. In this regard, we have shown how to achieve data isolation and to enable tenant-specific customizations. The additional development effort is quite large (Table 7) and therefore not recommended when operational cost benefits are not the priority. Instead of developing the multi-tenancy support in-house, it can be acquired from third parties. For example, Apprenda [5] is a private PaaS platform for .NET web applications on top of SQL server. It supports the execution of multi-tenant applications: it ensures data isolation and enables simple customizations, for example in the user interface.

Since the need for support for traditional enterprise applications in the cloud is increasing, PaaS category 1 can play a crucial role in facilitating the migration of these enterprise applications to the cloud. This also explains the high amount of platforms in this category compared to the other categories (Table 2). But SaaS providers could benefit more from economies of scale, if more development support would be provided to integrate with scalable cloud solutions as well as to endow applications with multi-tenancy.

PaaS category 2. The focused PaaS platforms are built on top of custom application servers, aiming for higher elasticity and scalability. However, this high level of scalability is achieved at the expense of portability. For example, APIs need to be adapted and optimized. Often these platforms target a specific type of cloud applications and offer some support for the development of such applications. For example, both Google App Engine and the XAP Elastic Application Platform by GigaSpaces [31] provide an abstraction layer on top of their scalable cloud storage to support JPA, though with limitations. Because of this focus on specific types of cloud applications, the set of supported technologies is limited, and different results for portability are obtained depending on which type of application is implemented. In the case of the hotel booking application on GAE, around $78 \%$ of the Java EE code could be reused, which seems quite acceptable.

With the rise of more established middleware providers in the PaaS field (Table 2), the PaaS platforms of category 2 try to extend their offerings to enhance portability. 
For example, we noticed that GAE gradually adds new Java classes to its white list and recently, Google added a MySQL database service to its offering (i.e. Google Cloud SQL). In addition, Google released a REST-based cloud storage API to access the datastore, thus improving integration. Both are still in beta phase. However, for each of these extensions, they still take non-functional requirements like scalability into account.

The focused PaaS platforms typically support data isolation for multi-tenant applications. We consider this to be a first step in the right direction. For example, GAE offers the Namespaces API to provide a multi-tenant database. However, development support for tenant-specific configuration and customization is lacking, so is a tenant-specific application management facility lacking as well. Similar to the platforms of category 1, the multi-tenancy support has to be custom developed, as illustrated in Section 5. Because a core requirement for multi-tenancy, i.e. data isolation, is already present, the additional development effort is less complex, but still substantial (Table 7).

PaaS category 3. The metadata-driven PaaS platforms, like Force.com, lack any compatibility with common programming models. This is mainly because, compared to PaaS categories 1 and 2, these platforms offer a composition and configuration interface at a higher abstraction layer. Even non-developers, who do not have any programming experience, could be able to build cloud applications by using a point-andclick interface to complete forms and models. Additional business logic and custom user interfaces are written using platform-specific programming/scripting languages. In our implementation, the development effort was acceptable (Table 7), because we tried to use the built-in features as much as possible, for example the standard web pages to access and manage the business objects (e.g. hotels and rooms) in the Force.com application. Only the booking process required the development of a custom user interface and corresponding controller (using VisualForce and Apex).

Moreover, this higher abstraction level limits the complexity of the applications and especially the processing logic that can be implemented. The lack of portability by the metadata-driven platforms is a core issue of this approach and cannot be bridged by the SaaS providers themselves. As the migration to PaaS platforms of this category requires a full rewrite, it inherently means that the application is also not easily portable to other PaaS platforms (even of the same category). Therefore, these platforms try to compensate it with extensive integration capabilities. With almost each new release, Force.com extended its set of toolkits to improve integration.

The metadata-driven PaaS platforms are the only PaaS category that offers fullfledged support for multi-tenancy, enabling tenants to customize SaaS applications to their preferences, even via custom code. It is remarkable that for example Salesforce.com, a company that is rather new in the role of middleware (platform) provider, beats the rest of the field in terms of multi-tenancy support. Yet the support for tenantspecific application management remains limited. For instance, Force.com does not support tenant-aware performance and resource consumption monitoring. 
7.2 Impact of case study application and implementation decisions

The choice of the online hotel booking application as a case study has an influence on the results of this experience report. So do the implementation decisions for the on-premise application. We discuss both elements below.

Impact of case study application. The online hotel booking application is a componentbased web application, that does not depend on external web services or applications. However, as discussed in Section 4, we did investigate the integration support provided by the different PaaS platforms to implement service compositions. Using another case study, consisting of a typical service composition, would therefore yield similar conclusions. (Yet it would enable us to illustrate how to solve the detected integration issues.)

Further, the data model of the hotel booking application contains several relationships between the different entities. Such a data model complicates the use of NoSQL datastores, as illustrated by the Windows Azure implementation using the Azure Table Storage. Also GAE imposes limitations on entity relationships. Blogs and surveys are typical examples of applications that are suitable for such key-value datastores, e.g. [49]. These applications have a simple data model with no or a limited amount of relationships between the entities. In the case of GAE and Azure Table Storage, we tried to solve this issue by applying a hierarchical data model with unidirectional relationships. As shown by the GAE implementation, this approach can work quite well. Therefore we think that the selection of the hotel booking application with a more complex data model, did not have a major impact on the results of portability. In addition, this decision resulted in the documentation of GAE offering better development support on top of the BigTable datastore compared to Azure with the Azure Table Storage, as the latter clearly focuses on structured data without entity relationships.

Impact of implementation decisions. We implemented two on-premise versions of the case study application (i.e. a Java EE and a .NET version), which we used as basis for the comparison of the three PaaS platforms with respect to the different requirements. The implementation decisions have especially impact on the portability requirement.

In the case of Force.com and other metadata-driven PaaS platforms, the selection of specific implementation technologies for the on-premise versions has no impact on the results. The metadata-driven architecture and the Salesforce.com-specific Apex language are incompatible with any common programming model or middleware framework, and therefore different implementation decisions do not improve the portability of the application.

The decision to use Enterprise JavaBeans (EJB) [25] in the Java version has a negative impact on the portability to GAE. GAE is focused on web applications and therefore the more heavy-weight component model of EJBs is not supported. However, the use of Java Servlets and JSPs for the front end of the application is in favour of GAE. For example, a front end consisting of Web Services would have shown worse results, because additional development is required for the handlers of SOAP 
messages. Consequently, we can claim that the mix of supported and unsupported technologies in the on-premise implementation generates a fair perspective on the portability support provided by GAE.

Portability is no issue for the platforms of category 1 and therefore our implementation decisions do not have a major impact on the results. Instead of the MVC framework, we could have easily used the Windows Communication Foundation (WCF) [48], a framework providing a unified platform for building service-oriented applications based on SOAP or the REST approach. The same applies if we had evaluated a Java-based PaaS from this category, e.g. Red Hat OpenShift [63].

\subsection{Challenges}

The main challenge that we extracted from the results of our work is the need to combine portability with operational cost efficiency (as shown in Fig. 6). None of the three categories of PaaS platforms sufficiently support both portability and development of multi-tenant SaaS applications. Currently, the focus of the existing platforms is on only one of these requirements. The challenge is to combine the best of two worlds: (i) maximum operational cost efficiency by supporting applicationlevel multi-tenancy, as well as (ii) portability across different platforms because of the use of mainstream programming models and middleware frameworks. We consider opportunities to address this challenge by architecting a middleware layer for application-level multi-tenancy and by standardization. We discuss this in depth in the following paragraphs, and identify several subchallenges concerning the different requirements based on the results of this experience report on developing multi-tenant SaaS applications on top of PaaS.

Middleware for application-level multi-tenancy. By applying multi-tenancy at the application level, resource sharing between the different tenants and thus operational efficiency are maximized. However, except for the metadata-driven platforms, current PaaS platforms hardly provide any support for developing multi-tenant applications. When needed, this support is currently implemented for each application separately. Ideally, the multi-tenancy concern is modularized into a reusable middleware service that can be enabled/disabled when necessary by the SaaS provider and configured to the application-specific requirements. This way, SaaS providers are able to easily endow their SaaS applications with multi-tenancy, without much development overhead.

A first requirement for such a middleware layer is the availability of development support for data isolation and tenant-specific customizations. We have explained how this can be achieved on top of GAE and Azure via dependency injection, but this approach should be generalized to reuse it for every application. A first step to prototype such a middleware has been achieved in [81]. In addition, more research is required to support the same level of flexibility in multi-tenant applications as the metadata-driven platforms (e.g. Force.com) on top of the PaaS platforms of the other categories. 
Besides development support for data isolation and customizable multi-tenant applications, major challenges still exist in achieving tenant-specific application management and performance isolation at runtime. None of the PaaS platforms that we investigated fully address these two requirements.

Tenant-specific application management requires the administration of SaaS applications to be isolated between the different tenants, for example to enable SaaS providers to monitor how many resources are consumed by a specific tenant (for billing and SLA compliance). In addition, this includes the need for APIs and tools to support application management, in particular for tenant-aware monitoring. Most of the PaaS platforms are limited to managing and monitoring complete applications or even VMs instead of on a per tenant basis. The monitored data is too coarsely-grained and not suitable for multi-tenant SaaS applications. This is clearly an open challenge that should be addressed by middleware as well as tool support.

Performance isolation is key to prevent one tenant from adversely affecting the performance of other tenants and to ensure that the performance of the different tenants comply with their service level agreements (SLAs) [36]. Typically, performance isolation is achieved by using virtualization (e.g. Windows Azure) or at the middleware level (e.g. GAE and Red Hat OpenShift). However, these approaches restrict resource sharing between different tenants and have a higher application management and maintenance cost. At the same time, more sharing complicates isolation between the tenants. Currently, SaaS providers are unable to guarantee any performancerelated SLAs to their tenants when hosting a multi-tenant SaaS application, even in the case of the metadata-driven platforms. Therefore, this complexity to achieve performance isolation in multi-tenant applications should also be tackled at the middleware level. In the meantime, SaaS providers have to make a trade-off between operational efficiency and fairness in the use of resources. This decision depends on the kind of application and the tenant-specific requirements. Moreover, it is possible to deploy a combination of multi-tenant and single-tenant applications to address the different requirements.

Standardization. Standardization is an appropriate solution to improve portability of SaaS applications across different on-premise and cloud-based platforms (public as well as private) and to tackle vendor lock-in. Based on our observations in this experience report, we believe that the need for standardization especially exist in (i) the use of non-relational storage and typical cloud services like caching, (ii) the support for multi-tenancy, and (iii) application management and monitoring. The latter two are strongly related to the challenges of a middleware layer for application-level multitenancy, as mentioned above. Standardization is especially relevant for the APIs provided by such a middleware layer. The issue with non-relational storage is illustrated with the Azure Table Storage, where we had to implement an application-specific persistence layer ourselves. If we decide to replace Azure Table Storage with an external cloud storage service, we would have to adapt this custom persistence layer to the new API. Some platforms already offer a persistence layer for non-relational storage (e.g. GAE and Amazon Elastic Beanstalk), but these are vendor-specific approaches and compromise the portability. 
In this regard, JSR 342, the Java EE 7 Specification [26], aims to enhance the suitability of the Java EE platform for cloud environments and can therefore be an interesting contribution to the standardization of Java-based PaaS platforms. The goal is to extend the Java EE platform with support for the PaaS model as well as a limited form of the SaaS model, while preserving as much as possible the current programming model. Concretely, the JSR proposes a.o. the following: (i) a definition of new platform roles for the PaaS model, (ii) metadata support for provisioning, QoS, isolation etc., (iii) potential standard APIs for non-relational databases, caching (JCache - JSR 107), management and monitoring, and (iv) multi-tenancy support in existing APIs, for example JPA. The latter is a first step to address the challenge of a middleware layer for application-level multi-tenancy, although it focuses mainly on data isolation. The proposed management and monitoring interfaces, however, are still at the granularity of complete applications (i.e. middleware-level multi-tenancy). The same applies to the specified isolation and QoS requirements.

With respect to integration support, standards exist in the form of SOAP- and REST-based web services, and as we explained, they are already commonly used by the different PaaS platforms. Only GAE did not have built-in support and required external libraries or additional development. Furthermore, more effort is required in providing development support and tools to improve integration with external cloud services (e.g. datastores) and platforms. This is important to tackle vendor lock-in. We notice that Force.com (with its toolkits), but especially the open source platforms are currently the front runners. An example of such a library is jclouds [41], an open source library that offers several API abstractions for cloud services like Amazon S3 and Azure Blob Storage. Finally, OCCI [56] comprises a set of specifications for cloud APIs to support integration, portability and interoperability. However, it focuses especially on IaaS.

\section{Related Work}

This section illustrates the gap in the current state of the art with respect to the indepth evaluation of cloud offerings based on practical case studies. The evaluation of cloud offerings has been limited to general comparisons between the different delivery models (i.e. IaaS, PaaS and SaaS), or discussions based on high-level concepts (e.g. computing architecture, storage and load balancing). In the context of IaaS, more in-depth studies surveys have been conducted; this paper is to the best of our knowledge the first of its kind in the growing space of PaS. Furthermore, this section discusses related work in the context of the main challenges that we identified based on our results: support for multi-tenancy in SaaS applications and portability across cloud platforms.

Surveys on cloud platforms. In the current state of the art, most research has been done in the context of IaaS with platforms such as Eucalyptus [55], OpenNebula [77], OpenStack [57] and CloudStack [21]. This results in surveys especially on IaaS platforms, e.g. [62]. Our work, however, focuses on PaaS platforms, and more specifically the development support for SaaS applications provided by these PaaS platforms. 
Armbrust et al. [6] distinguish different classes of utility computing based on the level of abstraction. At one end of the spectrum they classify Amazon EC2 [1], and at the other extreme of the spectrum application-domain-specific platforms such as Google App Engine [32]. Windows Azure [46] is an intermediate point on their spectrum. They compare how these three platforms virtualize computation, storage, and networking and how scalability and high availability is ensured. Further, the authors discuss the economics of cloud computing and what the opportunities and challenges are for cloud computing in general, including the issue of data lock-in (i.e. data portability across different platforms). The focus of their work is especially on runtime properties of cloud computing, while we compare PaaS platforms based on requirements related to the development of SaaS applications, i.e. portability, support for multi-tenancy, and tool support.

Other work, such as $[65,66,73]$, gives a general overview on cloud computing and discusses IaaS, PaaS as well as SaaS, including some examples. They are often limited to the enumeration of some high-level concepts (e.g. computing architecture, load balancing and storage), without any hands-on experience with the platforms. For example, the book "Enterprise Cloud Computing" [73] addresses cloud computing for enterprise applications, covering the common concepts and technologies like web services, virtualization and multi-tenancy. In addition, the author introduces several cloud platforms like Amazon EC2 [1], Google App Engine [32], and Windows Azure [46]. Furthermore, the work discusses extensively the concept of metadata-driven PaaS platforms, such as Force.com [67,82] and InstantApps [71]. These metadata-driven platforms are called Dev 2.0 platforms [72] by the author. We focus on an in-depth comparison of PaaS platforms (including the Dev 2.0 platforms) with respect to support for SaaS application development, on the basis of a case study that involves the development of multi-tenant SaaS applications on top of different PaaS platforms. Therefore, our work is complementary.

In [64], Reese explains how to design, build and maintain web applications that can be deployed into the cloud, including how to move existing web applications to the cloud. In addition, the book discusses differences between traditional on-premise deployment and cloud computing in areas such as reliability, security and scalability. However, the focus is on building applications on top of IaaS with Amazon Web Services (AWS) [2] as primary example, and the work does not tackle the issue of multi-tenancy at the application level. We, however, evaluate the development support for SaaS applications on top of several PaaS platforms, with support for multi-tenancy as one of the key criteria to achieve higher cost efficiency.

Support for multi-tenancy in SaaS applications. The state of the art on multi-tenancy in SaaS applications can be subdivided into multi-tenant data storage (i.e. multitenancy at the data tier), and methodologies and patterns to achieve multi-tenancy. Notice that multi-tenancy is only one of the requirements that we investigated with respect to SaaS application development.

A key requirement to support multi-tenancy in SaaS applications is data isolation between the different tenants. Chong et al. [20] identified three approaches for managing multi-tenant data and described a number of patterns to realize a multitenant data architecture. In [7], a new schema-mapping technique for multi-tenant 
databases, called Chunk Folding, is presented. [8] compares five techniques for implementing flexible schemas for multi-tenant SaaS applications. Our work compares PaaS platforms with respect to development support for multi-tenant SaaS applications, including data isolation. The schema-mapping techniques in the state of the art can be applied by PaaS providers to support data isolation in the platforms, or by SaaS providers to implement this support themselves when it is absent.

In [19], Chong and Carraro present the four-level SaaS maturity model: (i) singletenant, a separate custom application per tenant, (ii) configurable single-tenant, a separate instance of a configurable application for each tenant, (iii) configurable multitenant, a single instance serving every tenant with configurable metadata, and (iv) scalable, configurable multi-tenant, a load-balanced pool of identical multi-tenant instances, which are configurable to the tenant-specific requirements. Furthermore they describe a high-level architecture of a SaaS application, especially focusing on the addition of metadata services to manage the tenant-specific application configuration. Our work investigates the development of multi-tenant SaaS applications, customized to the tenant-specific requirements, on top of PaaS platforms. In principle, the PaaS platforms take care of the scalability concern. Therefore, our goal is to develop SaaS applications that correspond to level 4 in the SaaS maturity model (i.e. scalable, configurable multi-tenant).

Guo et al. [36] discuss the design and implementation principles to support the development of multi-tenant applications. The focus is on better isolation of security, performance, availability (i.e. fault isolation) and administration between the different tenants. Our work, however, focuses on the comparison of PaaS platforms, based on a practical case study, with respect to the provided development support for multitenant SaaS applications. The design principles presented by [36] can be applied in our work when the support for multi-tenancy by the PaaS platforms is inadequate.

Bezemer et al. [11] describe how to enable multi-tenancy in software services. They report their experiences with transforming an existing single-tenant software system into a multi-tenant one by means of their reengineering pattern [12]. This pattern requires three additional components: a multi-tenant database, tenant-specific authentication and configuration. The resulting SaaS application is hosted by the SaaS provider itself and is not deployed on top of a PaaS platform. In this paper, we compare the support offered by PaaS platforms to develop multi-tenant SaaS applications. One of our requirements regarding SaaS development is the need for multi-tenancy support. Therefore, the principles of the reengineering pattern complement our work and are used as requirements for the multi-tenancy support.

Portability across cloud platforms. Andrikopoulos et al. [4] analyze the migration of applications to the cloud based on a survey of the state of the art, identifying several challenges. A complete migration of an application to the cloud using the available cloud services is considered the most effective solution, however it requires the most re-engineering effort, showing the need for portability. In this work, we apply such a migration scenario and evaluate the support provided by PaaS platforms to develop SaaS applications based on a practical case study.

Mietzner et al. [50] propose an extension to the service component architecture (SCA) standard, adding variability descriptors and multi-tenancy patterns. This way, 
they try to combine portability with support for multi-tenancy. Similarly, [60] apply SCA to deploy applications on top of multiple heterogeneous PaaS and IaaS environments. Their federated PaaS infrastructure relies on their own FraSCAti application environment for SCA applications that should be deployed on top of each PaaS platform. In contrast to [50], their work does not offer support for multi-tenancy. However, SCA focuses on a limited set of application types, and applications can only be executed in a SCA-aware application environment (e.g. FraSCAti). In addition, SCA can only ensure portability with respect to computation. For example, it does not address the differences in the APIs of the different cloud services (e.g. storage, caching, etc.), which we identified as an important challenge in the current PaaS offerings.

The European mOSAIC project [61] develops a new platform with an open, independent API to support heterogeneous hybrid clouds. The goal is to deploy this platform on top of various existing PaaS platforms to enable the portability of componentbased applications that are developed for their (new) platform. The latter requirement, however, hinders the migration of existing, on-premise applications to this platform. Moreover, support for multi-tenancy is not addressed by this work.

\section{Conclusion}

To benefit from economies of scale, software vendors aim to migrate from an onpremise deployment model towards a Software-as-a-Service (SaaS) model. PaaS platforms aim to facilitate the development and deployment of cloud applications by delivering a computing platform and solution stack as a service. As with any paradigm shift, many new and somehow competing technologies enter the arena; more and more PaaS technologies have emerged. This evolution demands for independent research on the relative value and performance of new types of technologies. Given the broad spectrum of competing technologies, this paper addresses a selection of three platforms that each can be considered to be representative for a larger category of PaaS platforms.

The paper covers an in-depth investigation of the support of Force.com, Google App Engine and Windows Azure for the development of multi-tenant SaaS applications. The paper addresses these three representative PaaS platforms based on a practical application case study. Google App Engine and Windows Azure provide better portability based on their compatibility with the mainstream development model, but these platforms lack built-in support for multi-tenancy. Force.com introduces a metadata-driven architecture and an interpreted programming language (Apex) to support the development of multi-tenant applications that are tailored to tenant-specific requirements. However, this approach hinders straightforward portability. A major research and development challenge consists of providing the same level of flexibility for multi-tenant applications (similar to Force.com), but on PaaS platforms that apply a more traditional and therefore portable programming model.

Clearly, application-level multi-tenancy remains an important challenge as PaaS platforms offer insufficient support. Modularizing all aspects of the multi-tenancy requirement into a reusable middleware layer is an essential track of ongoing and future work. Finally, there is a need for standardization to improve portability of 
SaaS applications across different (cloud) platforms and to tackle and avoid vendor lock-in. Standardization is especially relevant for typical cloud services like scalable storage, and also to support application-level multi-tenancy.

Besides SaaS development on top of PaaS platforms, it is definitely worth and necessary to consider and evaluate other aspects of current PaaS offerings, for example operational aspects (e.g. monitoring, updating and patching), as well as business aspects (e.g. contracting and pricing). In addition, it will remain important to follow-up on the evolution and improving quality of existing PaaS offerings, also with respect to portability, multi-tenancy and tool support. This paper therefore offers but one, yet important, contribution to the experience reporting and analysis that is required to guide and support the paradigm shift and transition to SaaS.

Acknowledgements We thank the anonymous reviewers for their constructive comments to improve this paper. We also thank Wouter De Borger, Maarten Decat and Dimitri Van Landuyt for proofreading. This research is partially funded by the Research Fund KU Leuven, the iMinds CUSTOMSS project and by the EU FP7 project NESSoS. The iMinds CUSTOMSS is a project co-funded by iMinds (Interdisciplinary institute for Technology), a research institute founded by the Flemish Government. Companies and organizations involved in the project are Agfa Healthcare, Televic Healthcare and UnifiedPost, with project support of IWT (government agency for Innovation by Science and Technology).

\section{References}

1. Amazon Web Services LLC: Amazon Elastic Compute Cloud (Amazon EC2). http://aws . amazon. com/ec2/, Accessed 6 July 2013

2. Amazon Web Services LLC: Amazon Web Services (AWS). http://aws . amazon. com/, Accessed 6 July 2013

3. Amazon Web Services LLC: AWS Elastic Beanstalk. http://aws.amazon.com/ elasticbeanstalk/, Accessed 6 July 2013

4. Andrikopoulos, V., Binz, T., Leymann, F., Strauch, S.: How to Adapt Applications for the Cloud Environment. Computing pp. 1-43 (2012)

5. Apprenda Inc.: Apprenda - The Leading Private PaaS. http://apprenda.com/platform/, Accessed 6 July 2013

6. Armbrust, M., Fox, A., Griffith, R., Joseph, A.D., Katz, R.H., Konwinski, A., Lee, G., Patterson, D.A., Rabkin, A., Stoica, I., Zaharia, M.: Above the clouds: A berkeley view of cloud computing. Tech. Rep. 28, EECS Department, University of California, Berkeley (2009)

7. Aulbach, S., Grust, T., Jacobs, D., Kemper, A., Rittinger, J.: Multi-tenant databases for software as a service: schema-mapping techniques. In: SIGMOD '08: Proceedings of the ACM SIGMOD international conference on Management of Data. pp. 1195-1206. ACM, New York, NY, USA (2008)

8. Aulbach, S., Jacobs, D., Kemper, A., Seibold, M.: A comparison of flexible schemas for software as a service. In: SIGMOD '09: Proceedings of the ACM SIGMOD International Conference on Management of Data. pp. 881-888. ACM, New York, NY, USA (2009)

9. Azeez, A., Perera, S., Gamage, D., Linton, R., Siriwardana, P., Leelaratne, D., Weerawarana, S., Fremantle, P.: Multi-tenant SOA Middleware for Cloud Computing. In: IEEE International Conference on Cloud Computing. pp. 458-465. IEEE Computer Society, Los Alamitos, CA, USA (2010)

10. Baker, J., Bond, C., Corbett, J.C., Furman, J.J., Khorlin, A., Larson, J., Léon, J.M., Li, Y., Lloyd, A., Yushprakh, V.: Megastore: Providing Scalable, Highly Available Storage for Interactive Services. In: CIDR '11: Proceedings on Conference on Innovative Data Systems Research (2011)

11. Bezemer, C.P., Zaidman, A., Platzbeecker, B., Hurkmans, T., Hart, A.: Enabling Multi-Tenancy: An Industrial Experience Report. In: ICSM '10: Proceedings of the 26th International Conference on Software Maintenance. pp. 1-8 (2010)

12. Bezemer, C.P., Zaidman, A.: Challenges of Reengineering into Multi-Tenant SaaS Applications. Software Engineering Research Group (SERG) 12, TU Delft (2010) 
13. Bhuyan, L.N., Agrawal, D.P.: Generalized Hypercube and Hyperbus Structures for a Computer Network. Computers, IEEE Transactions on C-33(4), 323-333 (1984)

14. Bozman, J., Chen, G.: Cloud Computing: The Need for Portability and Interoperability. IDC Analyze the Future (August 2010), Sponsored by Red Hat, Inc.

15. Chang, F., Dean, J., Ghemawat, S., Hsieh, W.C., Wallach, D.A., Burrows, M., Chandra, T., Fikes, A. Gruber, R.E.: Bigtable: A Distributed Storage System for Structured Data. ACM Trans. Comput. Syst. 26(2), 4-1 (June 2008)

16. Chappell, D.: Introducing the Windows Azure Platform. http://www.davidchappell. com/writing/white_papers/Introducing_the_Windows_Azure_Platform,_v1. 4--Chappell.pdf (October 2010), Sponsored by Microsoft Corporation

17. Chen, P.P.S.: The Entity-Relationship Model - Toward a Unified View of Data. ACM Trans. Database Syst. 1(1), 9-36 (March 1976)

18. Chohan, N., Bunch, C., Pang, S., Krintz, C., Mostafa, N., Soman, S., Wolski, R.: AppScale: Scalable and Open AppEngine Application Development and Deployment. In: CloudComp '09: First International Conference on Cloud Computing. pp. 57-70. Springer Berlin Heidelberg (2010)

19. Chong, F., Carraro, G.: Architecture Strategies for Catching the Long Tail. Microsoft Corporation, http://msdn.microsoft.com/en-us/library/aa479069.aspx (April 2006)

20. Chong, F., Carraro, G., Wolter, R.: Multi-Tenant Data Architecture. Microsoft Corporation, http: //msdn.microsoft. com/en-us/library/aa479086. aspx (June 2006)

21. Citrix Systems, Inc.: CloudStack - Open Source Cloud Computing Project . http://cloudstack. org, Accessed 6 July 2013

22. CloudBees Inc.: CloudBees: The Java PaaS Company. http://www . cloudbees.com, Accessed 6 July 2013

23. Cordys: Cordys: The Enterprise Cloud Platform. http://www.cordys.com/ platform-as-a-service, Accessed 6 July 2013

24. DeMichiel, L.: JSR 317: Java ${ }^{T M}$ Persistence 2.0. http: //www . jcp. org/en/jsr/detail ?id=317 (2009), Accessed 6 July 2013

25. DeMichiel, L.: JSR 318: Enterprise JavaBeans ${ }^{T M}$ 3.1. http://jcp.org/en/jsr/detail?id=318 (2010), Accessed 6 July 2013

26. DeMichiel, L., Shannon, B.: JSR 342: Java ${ }^{T M}$ Platform, Enterprise Edition 7 (Java EE 7) Specification. http: //www.jcp.org/en/jsr/detail?id=342 (2011), Accessed 6 July 2013

27. Engelsen, N.: Multi Tenant Architecture via Dependency Injection. http://blog.tallan.com/ 2010/07/11/multi-tenant-architecture-via-dependency-injection-part-1/ (July 2010), Accessed 6 July 2013

28. Fowler, M.: Inversion of Control Containers and the Dependency Injection pattern. http:// martinfowler.com/articles/injection.html (January 2004)

29. Garen, K.: Software Portability: Weighing Options, Making Choices. The CPA Journal 77(11), 10-12 (2007)

30. Giessmann, A., Stanoevska-Slabeva, K.: Platform as a Service : A Conjoint Study on Consumers' Preferences. In: ICIS '12: 33rd International Conference on Information Systems. Association for Information Systems (AIS) (2012)

31. GigaSpaces Technologies, Inc.: XAP - Elastic Application Platform. http://www.gigaspaces . com/xap/, Accessed 6 July 2013

32. Google, Inc.: Google App Engine. http://code.google.com/appengine/, Accessed 6 July 2013

33. Google Inc.: Guice. http: //code.google.com/p/google-guice/, Accessed 6 July 2013

34. Google, Inc.: The JRE Class White List. http://code.google.com/appengine/docs/java/ jrewhitelist.html, Accessed 6 July 2013

35. Google, Inc.: Will it play in Java. http://code.google.com/p/googleappengine/wiki/ WillItPlayInJava, Accessed 6 July 2013

36. Guo, C.J., Sun, W., Huang, Y., Wang, Z.H., Gao, B.: A framework for native multi-tenancy application development and management. In: CEC/EEE '07: 9th IEEE International Conference on E-Commerce Technology and 4th IEEE International Conference on Enterprise Computing, E-Commerce, and EServices. pp. 551-558 (July 2007)

37. Heroku, Inc.: Heroku Cloud Application Platform. http://www.heroku.com/, Accessed 6 July 2013

38. Hillyer, S.: An Introduction to Environments. http://wiki.developerforce.com/index.php/ An_Introduction_to_Environments, Accessed 6 July 2013

39. Hiranandani, S., Kennedy, K., Tseng, C.W.: Compiling Fortran D for MIMD distributed-memory machines. Commun. ACM 35(8), 66-80 (1992) 
40. IBM Corporation: IBM SmartCloud Application Services. http://www.ibm.com/ cloud-computing/us/en/paas.html, Accessed 6 July 2013

41. jclouds, Inc.: jclouds: Cloud Interfaces, Simplified. http://www.jclouds.org/, Accessed 6 July 2013

42. Kohari, N.: Ninject! Open Source Dependency Injector for .NET. http://ninject.org/, Accessed 6 July 2013

43. Mell, P., Grance, T.: The NIST Definition of Cloud Computing. Special Publication 800-145, National Institute of Standards and Technology (NIST) (September 2011), http://csrc.nist.gov/ publications/nistpubs/800-145/SP800-145.pdf

44. Microsoft Corp.: ADO.NET Entity Framework. http://msdn.microsoft.com/en-us/data/ aa937723, Accessed 6 July 2013

45. Microsoft Corp.: ASP.NET MVC. http://www. asp.net/mvc, Accessed 6 July 2013

46. Microsoft Corp.: Windows Azure. http://www.windowsazure.com/, Accessed 6 July 2013

47. Microsoft Corp.: Windows Azure AppFabric. http://www.microsoft.com/windowsazure/ AppFabric, Accessed 6 July 2013

48. Microsoft Corp.: Windows Communication Foundation (WCF). http://msdn.microsoft.com/ en-us/netframework/aa663324, Accessed 6 July 2013

49. Microsoft Corp.: Windows Azure Architecture Guide. http://wag.codeplex.com/ (2010), Accessed 6 July 2013

50. Mietzner, R., Leymann, F., Papazoglou, M.P.: Defining Composite Configurable SaaS Application Packages Using SCA, Variability Descriptors and Multi-tenancy Patterns. In: ICIW '08: Third International Conference on Internet and Web Applications and Services. pp. 156-161 (June 2008)

51. Mooney, J.D.: Strategies for supporting application portability. Computer 23(11), 59-70 (nov 1990)

52. Mordani, R.: JSR 315: Java ${ }^{T M}$ Servlet 3.0 Specification. http://www . jcp.org/en/jsr/detail? id=315 (2009), Accessed 6 July 2013

53. Natis, Y.V., Smith, D.M., Cearley, D.W.: Windows Azure AppFabric: A Strategic Core of Microsoft's Cloud Platform. http://www.gartner.com/id=1469531 (November 2010)

54. Noelios Technologies: Restlet. http://www.restlet.org/, Accessed 6 July 2013

55. Nurmi, D., Wolski, R., Grzegorczyk, C., Obertelli, G., Soman, S., Youseff, L., Zagorodnov, D.: The Eucalyptus Open-Source Cloud-Computing System. In: CCGRID '09: Proceedings of the 9th IEEE/ACM International Symposium on Cluster Computing and the Grid. pp. 124-131. IEEE Computer Society, Washington, DC, USA (2009)

56. Open Grid Forum (OGF): Open Cloud Computing Interface. http://occi-wg.org/, Accessed 6 July 2013

57. OpenStack, LLC: OpenStack Open Source Cloud Computing Software. http://www. openstack. org/, Accessed 6 July 2013

58. Oracle Corporation: Jersey. http://jersey · java.net/, Accessed 6 July 2013

59. Oracle Corporation: Oracle Cloud Platform. https://cloud.oracle.com/mycloud/f?p= service: java:0, Accessed 6 July 2013

60. Paraiso, F., Haderer, N., Merle, P., Rouvoy, R., Seinturier, L.: A Federated Multi-cloud PaaS Infrastructure. In: CLOUD '12: IEEE 5th International Conference on Cloud Computing. pp. 392-399 (June 2012)

61. Petcu, D.: Portability and Interoperability between Clouds: Challenges and Case Study. In: Abramowicz, W., Llorente, I., Surridge, M., Zisman, A., Vayssière, J. (eds.) ServiceWave '11: Towards a Service-Based Internet. pp. 62-74. Springer Berlin Heidelberg (2011)

62. Prodan, R., Ostermann, S.: A survey and taxonomy of infrastructure as a service and web hosting cloud providers. In: 10th IEEE/ACM International Conference on Grid Computing. pp. 17-25 (oct 2009)

63. Red Hat, Inc.: Red Hat OpenShift. https : //openshift.redhat. com/, Accessed 6 July 2013

64. Reese, G.: Cloud Application Architectures. Theory in Practice, O'Reilly (2009)

65. Rimal, B.P., Choi, E., Lumb, I.: A Taxonomy and Survey of Cloud Computing Systems. In: NCM '09: 5th International Joint Conference on INC, IMS and IDC. pp. 44-51 (aug 2009)

66. Rosenberg, J., Mateos, A.: The Cloud at Your Service. Manning Pubs Co Series, Manning Publications (2010)

67. Salesforce.com, Inc.: Force.com. http://www.salesforce.com/platform/, Accessed 6 July 2013

68. Salesforce.com, Inc.: Salesforce CRM. http://www. salesforce.com/, Accessed 6 July 2013

69. SAP AG: SAP NetWeaver. http://www.sap.com/platform/netweaver/components, Accessed 6 July 2013 
70. Schubert, L., Jeffery, K., Neidecker-Lutz, B.: The Future of Cloud Computing: Opportunities for European Cloud Computing Beyond 2010. Expert Group Report, Public Version 1.0, European Commission, Information Society and Media (January 2010)

71. Shroff, G., Agarwal, P., Devanbu, P.: InstantApps: A WYSIWYG model driven interpreter for web applications. In: ICSE-Companion '09: 31st International Conference on Software Engineering - Companion Volume . pp. 417-418 (May 2009)

72. Shroff, G.: Dev 2.0: model driven development in the cloud. In: SIGSOFT '08/FSE-16: Proceedings of the 16th ACM SIGSOFT International Symposium on Foundations of software engineering. pp. 283-283. ACM, New York, NY, USA (2008)

73. Shroff, G.: Enterprise Cloud Computing: Technology, Architecture, Applications. Cambridge University Press (2010)

74. Siegel, H.J.: Interconnection Networks for SIMD Machines. Computer 12(6), 57-65 (1979)

75. Singh, J.P., Weber, W.D., Gupta, A.: SPLASH: Stanford parallel applications for shared-memory. SIGARCH Comput. Archit. News 20(1), 5-44 (1992)

76. Snir, M., Otto, S.W., Walker, D.W., Dongarra, J., Huss-Lederman, S.: MPI: The Complete Reference. MIT Press, Cambridge, MA, USA (1995)

77. Sotomayor, B., Montero, R.S., Llorente, I.M., Foster, I.: Virtual Infrastructure Management in Private and Hybrid Clouds. IEEE Internet Computing 13(5), 14-22 (sept-oct 2009)

78. Sun, W., Zhang, X., Guo, C.J., Sun, P., Su, H.: Software as a Service: Configuration and customization perspectives. In: SERVICES-2 '08: IEEE Congress on Services Part II. pp. 18-25 (Sept 2008)

79. Vaquero, L.M., Rodero-Merino, L., Caceres, J., Lindner, M.: A break in the clouds: towards a cloud definition. SIGCOMM Comput. Commun. Rev. 39(1), 50-55 (January 2009)

80. VMware, Inc.: Cloud Foundry. http://www . cloudfoundry. org/, Accessed 6 July 2013

81. Walraven, S., Truyen, E., Joosen, W.: A middleware layer for flexible and cost-efficient multi-tenant applications. In: Middleware '11: Proceedings of the 12th ACM/IFIP/USENIX International Conference on Middleware. pp. 370-389. Springer Berlin / Heidelberg (2011)

82. Weissman, C.D., Bobrowski, S.: The design of the force.com multitenant internet application development platform. In: SIGMOD '09: Proceedings of the 35th SIGMOD international conference on Management of data. pp. 889-896. ACM, New York, NY, USA (2009)

83. Wolf Frameworks India Pvt. Ltd.: WOLF. http://www.wolfframeworks.com/overview.asp, Accessed 6 July 2013

84. $\mathrm{WSO}_{2}$ Inc.: WSO2 Stratos. http://wso2.com/cloud/stratos/, Accessed 6 July 2013 